\title{
Perfil de pesquisa sobre gerenciamento de riscos em cadeias de suprimentos
}

\section{Analysis of supply chain risk management researches}

\author{
Ualison Rébula de Oliveira ${ }^{1,2}$ \\ Luciano Souza Espindola ${ }^{3}$ \\ Fernando Augusto Silva Marins ${ }^{4}$
}

\begin{abstract}
Resumo: Nos últimos 10 anos, publicações sobre Gerenciamento de Riscos em Cadeias de Suprimentos (SCRM) cresceram a uma taxa média de mais de $40 \%$ ao ano. Trata-se de um tema que vem, rapidamente, ganhando notoriedade na área de Logística, tanto por ser um assunto relativamente novo, inspirando pesquisadores a desenvolverem estudos sobre a questão, quanto pelo potencial de minimizar prejuízos de altas cifras em cadeias de suprimentos. Dada a relevância científica e empresarial da matéria, a presente pesquisa possui como objetivo principal analisar o perfil dos trabalhos publicados sobre SCRM, ou seja, mapear o campo de pesquisas sobre o tema, abrangendo os artigos publicados sobre esse assunto até 31 de dezembro de 2015. Para a consecução desse objetivo, adotou-se o método de pesquisa Research Profiling, que amplia o escopo da bibliometria por meio da mineração de texto. Para a análise, tabulação e organização dos dados, utilizou-se o software VantagePoint ${ }^{\circledast}$. Como resultados principais, foram respondidas inúmeras questões relacionadas às pesquisas sobre SCRM, das quais se destacam as seguintes: i) cada artigo, apresenta-se, em média, repetido 1,2 vez em outras bases de dados; ii) os autores mais citados não são os que mais publicam; iii) as palavras-chave "Supply Chain Risk Management" só se tornou representativa para esse tema após 2012; iv) apenas três periódicos, juntos, são responsáveis por um terço de todas as citações em publicações sobre SCRM. Como resultado secundário, mas não menos importante, observou-se a falta de consenso entre os pesquisadores quando o assunto diz respeito às etapas que devem ser desenvolvidas no SCRM.
\end{abstract}

Palavras-chave: Supply Chain Risk Management; SCRM; Perfil de pesquisa; Revisão sistemática da literatura.

\begin{abstract}
Despite the large number of contributions (some 250 articles) published on Supply Chain Risk Management (SCRM), none of them have developed what can be called research profiling on the theme. This paper aims to analyze the profile of works published on SCRM, that is, to map the field of research on the theme, covering articles published from 2004 to 2015. The authors adopted the research profiling method, which expands the scope of bibliometry by applying text mining. The VantagePoint ${ }^{\circledR}$ software was used to analyze, classify, and organize the data of this study. The results provide several insights into research on SCRM, namely: (i) the key expression "supply chain risk management" only became representative for the theme after 2012; (ii) the most cited authors are not the same as those who have published the most; and (iii) only three periodicals together account for one-third of all the citations in publications on SCRM.
\end{abstract}

Keywords: Supply Chain Risk Management; SCRM; Research profiling; Systematic literature review.

\section{Introdução}

Conforme Ghadge et al. (2012), as fontes de risco são numerosas e originam-se na própria empresa ou em seu ambiente de negócios, motivo pelo qual, segundo Christopher \& Lee (2004), a Gestão de Riscos está se tornando parte integrante de um projeto holístico da Gestão da Cadeia de Suprimentos (Supply Chain Risk Management - SCRM).

Colicchia \& Strozzi (2012) ressaltam que os riscos operacionais não são os únicos tipos de risco existentes em uma cadeia de suprimentos, uma vez

\footnotetext{
${ }^{1}$ Programa de Pós-graduação em Administração - PPGA, Universidade Federal Fluminense - UFF, CEP 27213-145, Volta Redonda, RJ, Brasil, e-mail: ualison.oliveira@gmail.com

${ }^{2}$ Laboratório de Tecnologia, Gestão de Negócios e Meio Ambiente - LATEC, Universidade Federal Fluminense - UFF, CEP 27213-145, Volta Redonda, RJ, Brasil

${ }^{3}$ Universidade Federal Fluminense - UFF, CEP 27213-145, Volta Redonda, RJ, Brasil, e-mail: lucianosouza1993@gmail.com

${ }^{4}$ Departamento de Produção, Faculdade de Engenharia - FEG, Universidade Estadual Paulista Júlio de Mesquita Filho - UNESP, CEP 12516-410, Guaratinguetá, SP, Brasil, e-mail: fmarins@feg.unesp.br
}

Recebido em Out. 14, 2016 - Aceito em Fev. 13, 2017

Suporte financeiro: nenhum. 
que a incerteza do ambiente de negócios e a própria complexidade das redes de cadeia de suprimentos estão aumentando a probabilidade de sua ruptura. Nesse contexto, Hendricks et al. (2009) advogam que, ao gerenciar esse tipo de risco, minimizam-se problemas com interrupções, reduz-se o seu impacto negativo sobre o desempenho e propicia-se a celeridade da restauração da cadeia de suprimentos para seu estado normal.

Problemas no Gerenciamento da Cadeia de Suprimentos (Supply Chain Management - SCM) podem gerar prejuízos de alto valor monetário, a exemplo do que ocorreu na década passada com as empresas Boeing, Cisco e Pfizer, que amargaram perdas de U\$ 2 bilhões, U\$ 2,25 bilhões e U\$ 2,8 bilhões, respectivamente (Hult et al., 2010). Outros exemplos de perdas financeiras ocasionadas por rupturas em cadeias de suprimentos podem ser encontradas em Kern et al. (2012) e Sodhi et al. (2012).

Segundo Colicchia \& Strozzi (2012), poucas áreas de interesse no âmbito da gestão ganharam tamanha notoriedade nos últimos anos como a SCRM, tanto do ponto de vista prático como de pesquisa.

O conceito de SCRM surgiu como uma extensão natural do SCM e se origina da intersecção entre a Gestão de Riscos e a Gestão da Cadeia de Suprimentos (Blos et al., 2009), conforme ilustra a Figura 1.

O SCRM emerge como um importante processo no SCM, com o objetivo principal de identificar as fontes potenciais de risco, sugerir medidas adequadas para mitigá-los (Singhal et al., 2011) e aumentar a resiliência da cadeia de suprimentos (Pujawan \& Geraldin, 2009). A Figura 2, ilustra o crescimento de publicações de pesquisa sobre SCRM em periódicos internacionais, nas bases de dados Scopus, Web of Science, Science Direct, Emerald Insight e Ingenta Connect.

Apesar das inúmeras contribuições dos cerca de 250 trabalhos publicados sobre SCRM até o final de

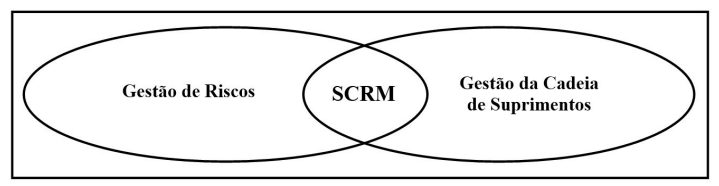

Figura 1. SCRM como a intersecção entre SCM e gestão de riscos. Fonte: Blos et al. (2009).

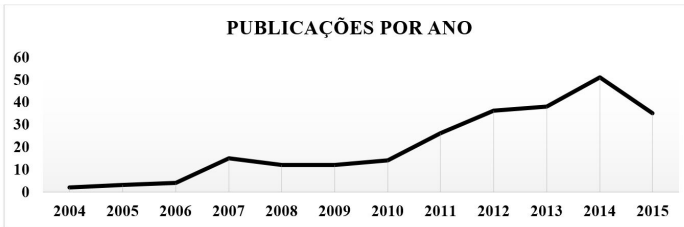

Figura 2. Publicações anuais sobre SCRM nas bases de dados selecionadas. Fonte: elaboração dos autores.
2015, verificou-se que nenhum deles desenvolveu um Research Profiling (Perfil de Pesquisa) sobre o tema. Os autores Tang (2006), Khan \& Burnes (2007) e Manuj \& Mentzer (2008b) fizerem extensas revisões daquilo que havia sido publicado sobre SCRM, contribuindo para a identificação de lacunas e com desenvolvimento de uma agenda de pesquisa. Colicchia \& Strozzi (2012) combinaram a Revisão Sistemática de Literatura (Systematic Literature Review - SLR) e a Análise de Redes de Citação (Citation Network Analysis - CNA) para investigar o processo de criação, transferência e desenvolvimento de conhecimento sobre o SCRM. Para tanto, eles analisaram 55 trabalhos oriundos da base de dados Web of Science, até 2010.

Dessa forma, a presente pesquisa busca complementar os trabalhos de revisão de literatura anteriormente descritos, utilizando como insumo todos os artigos publicados nas bases de dados Scopus, Web of Science, Science Direct, Emerald Insight e Ingenta Connect. Para tanto, selecionlu-se o método de Perfil de Pesquisa (Research Profiling), que amplia a revisão da literatura à medida em que examina as publicações de forma mais completa (Porter et al., 2002).

O Perfil de Pesquisa procura responder quatro perguntas: "Quem", "O quê", "Onde" e "Quando". Por exemplo: Quem são os autores mais citados em determinada área? O que é mais estudado dentro desse tema? Onde são produzidos mais artigos? Quando cada tópico aparece na literatura? O uso dessas perguntas não restringe a amplitude desse método, podendo ser construídas outras análises, tais como: quais países produzem mais pesquisa sobre determinado tópico? Quais as palavras-chave mais utilizadas por pesquisadores da área? Como se deu a evolução do volume de publicações ao longo do tempo?

No que tange a relevância deste trabalho, julga-se ser a mesma importante por pelo menos três aspectos: (i) pesquisas sobre SCRM ainda estão em estágio embrionário (Lavastre et al., 2012; Narasimhan \& Talluri, 2009), principalmente em países como o Brasil (Blos et al., 2009); (ii) pesquisas sobre SCRM estão, rapidamente, ganhando notoriedade na área de Logística (Colicchia \& Strozzi, 2012; Wieland \& Wallenburg, 2012; Singhal et al., 2011); (iii) os prejuízos por problemas na cadeia de suprimentos podem chegar a grandes cifras (Hult et al., 2010; Kern et al., 2012; Sodhi et al. 2012).

Quanto à estrutura deste trabalho: na seção 2 abordam-se questões metodológicas; a seção seguinte apresenta os resultados obtidos na pesquisa e uma discussão sobre eles; a seção 4 aborda perspectivas de avanços em pesquisas sobre SCRM e, finalizando o trabalho, encontram-se as principais conclusões, seguidas das referências bibliográficas utilizadas. 


\section{Aspectos metodológicos da pesquisa}

Nesta pesquisa fez-se uma SLR sobre SCRM em cinco diferentes bases de dados, utilizando-se bibliometria e mineração de texto para o desenvolvimento de um perfil de pesquisa, denominada por Porter et al. (2002) de Research Profiling.

A primeira etapa da pesquisa identificou todos os artigos publicados até 31/12/2015. Essa busca de dados ocorreu em 14 de março de 2016, por meio da opção pesquisa avançada em cada uma das bases de dados selecionadas, usando-se como argumento de pesquisa a expressão "Supply Chain Risk Management" no título, resumo e palavras-chave. Além disso, para fins de refinamento da pesquisa, selecionaram-se apenas artigos oriundos de periódicos científicos em língua inglesa.

O número de artigos encontrados para cada base de dados encontra-se na Tabela 1.

Como fatores limitadores dessa primeira etapa, destacam-se dois: (i) periódicos que ainda não haviam publicado a última edição de 2015 até 14/3/2016 não foram incluídos na pesquisa; (ii) pesquisas que não utilizaram a expressão "Supply Chain Risk Management" no título, palavras-chave ou resumo ao menos uma vez não foram selecionadas;

Tabela 1. Número de artigos encontrados por base de dados.

\begin{tabular}{cc}
\hline Base de dados & Número de artigos \\
\hline Scopus & 220 \\
Web of Science & 139 \\
Ingenta Connect & 66 \\
Emerald Insight & 63 \\
Science Direct & 55 \\
TOTAL & $\mathbf{5 4 3}$ \\
\hline
\end{tabular}

Fonte: elaboração dos autores.
A segunda etapa foi definir a forma pela qual esses dados seriam analisados. Para tanto selecionou-se o software VantagePoint ${ }^{\circledR}$. Justifica-se a escolha desse software por ele ser reconhecido por alguns pesquisadores pela eficiência e praticidade no tratamento dos dados (Eldridge, 2006; Islam \& Miyazaki, 2010; Kim et al., 2012).

A terceira etapa da pesquisa consistiu na organização dos dados para serem introduzidos no software VantagePoint ${ }^{\circledR}$. As bases Web of Science e Scopus geram arquivos que contêm todas as informações necessárias para a consecução do Research Profiling, bastando tão somente inseri-los no software em questão. As demais bases, Science Direct, Ingenta Connect e Emerald Insight, não fornecem todas as informações de forma automática, o que demandou a necessidade de analisar cada artigo dessas bases individualmente, com a posterior organização manual dos dados coletados.

Durante a terceira etapa da pesquisa, observou-se a existência de interseções (sobreposições) entre as bases, ou seja, um mesmo artigo estava presente em mais de uma base de dados. A Figura 3 resume essas constatações.

A Figura 3 permite verificar que a maior parte dos artigos sobre SCRM publicados nas bases Science Direct, Ingenta Connect e Emerald Insight estão presentes nas bases Web of Science e Scopus. Além disso, a Figura 3 ilustra que há uma interseção de 107 artigos entre as duas maiores bases (Scopus e Web of Science). Por fim, constata-se que: (i) $14 \%$ de todos os artigos da Ingenta Connect e $8 \%$ dos artigos da Emerald Insight não estão nas bases Scopus e Web of Science; (ii) que todos os artigos (100\%) da Science Direct estão presentes nas bases Scopus e/ou Web of Science.

Logo, para a pesquisa realizada foram usados os arquivos de dados gerados "automaticamente" pelas bases Web of Science e Scopus, mais uma matriz criada

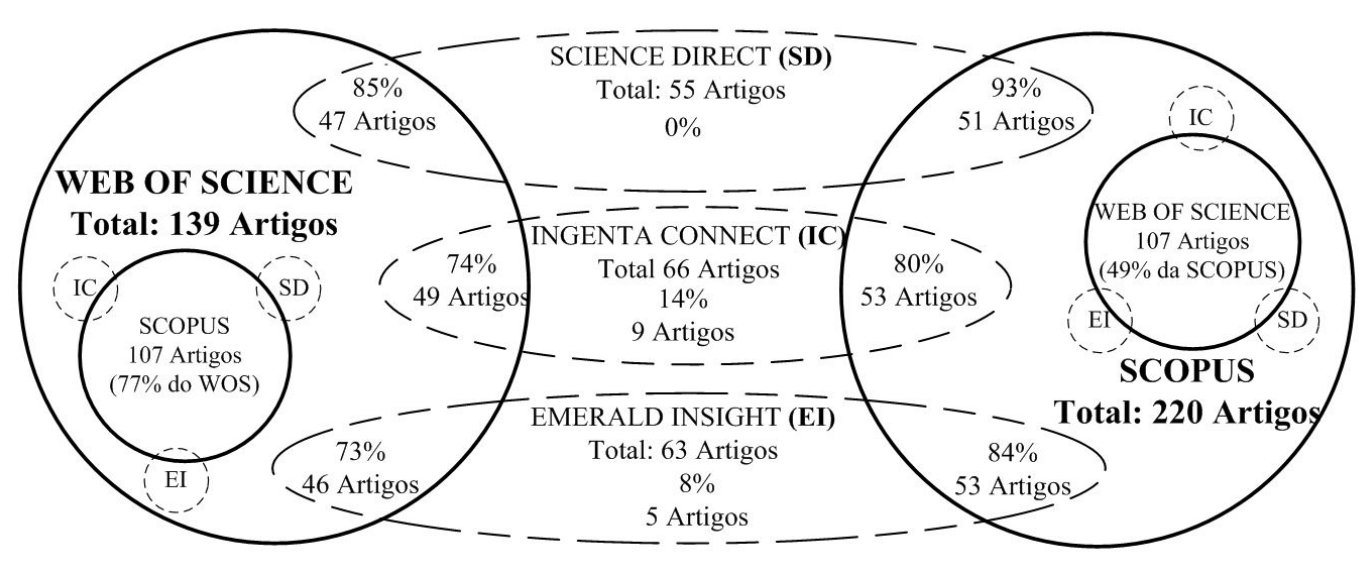

Figura 3. Intersecções entre as bases de dados para publicações sobre SCRM no período 2004-2015. Fonte: elaboração dos autores. 
manualmente com dados dos artigos pertencentes exclusivamente às bases Ingenta Connect ( 9 artigos) e Emerald Insight (5 artigos). Por fim, com todos os dados inseridos no VantagePoint ${ }^{\circledR}$ foi possível identificar a duplicidade de 107 artigos que eram comuns aos arquivos gerados pela Web of Science e Scopus. Após eliminação desses 107 registros, 248 artigos permaneceram como material de trabalho para o Research Profiling sobre SCRM, ou seja, $45,86 \%$ dos 543 artigos inicialmente levantados nas cinco bases de dados pesquisadas. Com base nesse material iniciou-se a quarta etapa da pesquisa.

A quarta etapa foi responsável pela organização e compatibilização dos dados dos artigos, quando foram padronizados nome dos periódicos, dos autores e países e palavras-chave, além dos outros itens considerados na análise. Essa ação foi realizada também por meio da utilização do software VantagePoint ${ }^{\circledR}$.

Na quinta e última etapa da pesquisa, realizaram-se análises básicas para identificar informações acerca dos dados organizados pelo software e, em seguida, análises avançadas para observar os padrões de produção de conhecimento no tema SCRM. Tais análises tiveram por propósito responder a seguinte questão: como se dá a dinâmica de produção de conhecimento na área de Gerenciamento de Riscos em Cadeias de Suprimentos?

Finalizando o trabalho, buscou-se definir e responder, as perguntas de interesse:

1. Quem são os autores mais produtivos?

2. Quais são os periódicos que mais publicam artigos na área estudada?

3. Quais as instituições mais produtivas?

4. Quais os países mais representativos na produção da área?

5. Quem são os autores mais referenciados?

6. Quais são os periódicos mais referenciados?

7. Em quais anos houve maior número de citações?

8. Quando houve maior volume de publicações na área em questão?

9. Quais as palavras-chave mais utilizadas no campo de pesquisa alvo?

Na próxima seção estão os resultados das cinco etapas desta pesquisa.

\section{Resultados do perfil de pesquisa sobre SCRM}

Essa seção organiza os resultados do Research Profiling sobre o tema SCRM. Uma vez que o software VantagePoint ${ }^{\circledR}$ apresenta resultados relacionados ao número de registros e/ou ao número de instâncias, ressalta-se que todas as figuras e tabelas foram ordenadas pelo número de registros fornecidos. Para uma melhor compreensão do significado dessa observação, tome-se o seguinte exemplo: um único artigo pode referenciar cinco obras de um mesmo autor; dessa forma, ter-se-ia um registro e cinco instâncias. As análises são apresentadas a seguir, em três subgrupos.

\subsection{Subgrupo "Mais publicam"}

Aqui busca-se responder a 4 questões, sendo que 3 delas estão representadas na Figura 4, onde se observa uma relação de dependência, relacionamento e interação entre elas.

Os autores que mais publicam sobre SCRM acabam por influenciar nos resultados bibliométricos das instituições e países que mais publicam sobre esse tema. A Tabela 2 inclui as 10 instituições que mais publicaram até 2015 .

Entretanto, essa relação não é proporcional, pois para vários pesquisadores suas publicações estão divididas entre as instituições em que estão ou estiveram trabalhando. Além disso, autores (diversos) que publicaram diferentes trabalhos estando na mesma instituição, acabam por aumentar a quantidade de publicações daquela instituição. A Tabela 3 relaciona os pesquisadores que mais publicaram sobre SCRM até 2015.

E a Figura 5 aborda a evolução das publicações desses autores ao longo dos anos.

Sobre os países que mais publicaram sobre SCRM, observa-se que Estados Unidos e China são líderes em termos de número de publicações, conforme ilustrado na Tabela 4.

\subsection{Subgrupo "Mais citados"}

Aqui busca-se responder perguntas relacionadas à quantidade de citações que autores e periódicos tiveram, bem como identificar os anos mais citados

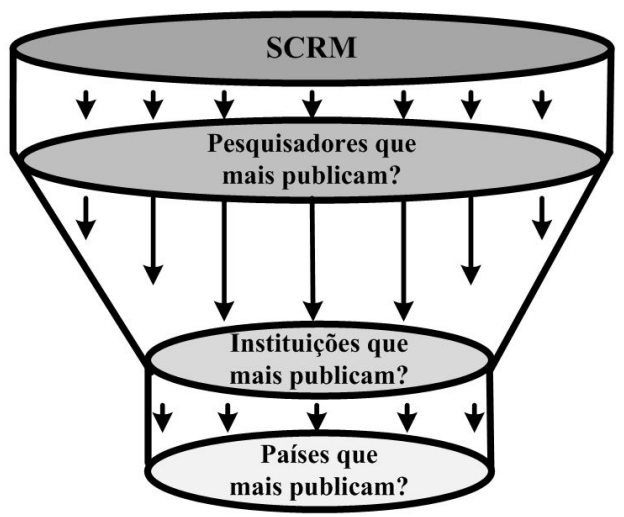

Figura 4. Relações entre autores, instituições e países que mais publicam sobre SCRM. Fonte: elaboração dos autores. 
Tabela 2. Relação das instituições que mais publicaram sobre SCRM.

\begin{tabular}{ccc}
\hline Ordenação & Instituição & Número de registros \\
\hline 1 & University of North Texas & 5 \\
\hline 2 & Cranfield School of Management & 4 \\
\hline 3 & Swiss Federal Institute of Technology & 4 \\
\hline 4 & University of Toronto & 4 \\
\hline 5 & Sepuluh Nopember Institute of Technology & 3 \\
\hline 6 & Hong Kong Polytechnic University & 3 \\
\hline 7 & Indian Institute of Technology Delhi & 3 \\
\hline 8 & Iowa State University & 3 \\
\hline 9 & University of Electronic Science and Technology of China & 3 \\
\hline 10 & Massachusetts Institute of Technology & 2 \\
\hline
\end{tabular}

Fonte: elaboração dos autores.

Tabela 3. Relação dos 20 autores que mais publicaram sobre SCRM.

\begin{tabular}{ccc}
\hline Ordenação & Autor & Número de registros \\
\hline 1 & Backhurst, J. & 6 \\
\hline 2 & Samvedi, A. & 5 \\
\hline 3 & Wagner, S. M. & 4 \\
\hline 4 & Ekwall, D. & 4 \\
\hline 5 & Khan, O. & 4 \\
\hline 6 & Manuj, I. & 4 \\
\hline 7 & Olson, D. L. & 3 \\
\hline 8 & Wu, D. & 3 \\
\hline 9 & Bandaly, D. & 3 \\
\hline 10 & Banwet, D. K. & 3 \\
\hline 11 & Chen, H. & 3 \\
\hline 12 & Christopher, M. & 3 \\
\hline 13 & Dani, S. & 3 \\
\hline 14 & Devadasan, S. R. & 3 \\
\hline 15 & Elangovan, D. & 3 \\
\hline 16 & Faisal, M. N. & 3 \\
\hline 17 & Goh, M. & 3 \\
\hline 18 & Jain, V. & 3 \\
\hline 19 & Karuppuswamy, P. & 3 \\
\hline 20 & Mentzer, J. T. & 4 \\
\hline
\end{tabular}

Fonte: elaboração dos autores.

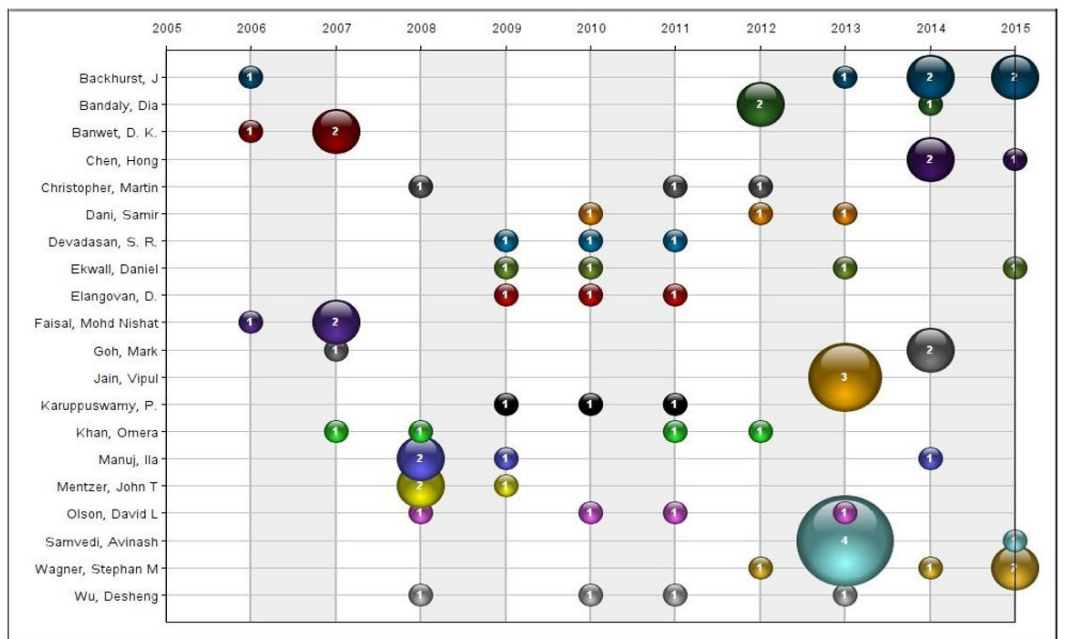

Figura 5. Artigos publicados por autor sobre SCRM até 2015. Fonte: elaboração dos autores a partir do software VantagePoint ${ }^{\circledR}$. 
pelos pesquisadores. A Tabela 5 organiza os 20 autores mais citados no desenvolvimento de pesquisas sobre o tema SCRM.

Já a Figura 6, aborda a evolução dos autores mais citados no período estudado.

Tabela 4. Relação dos 20 países que mais publicaram sobre SCRM.

\begin{tabular}{ccc}
\hline Ordenação & Países & $\begin{array}{c}\text { Número de } \\
\text { registros }\end{array}$ \\
\hline 1 & USA & 69 \\
\hline 2 & China & 37 \\
\hline 3 & Índia & 26 \\
\hline 4 & Grã-Bretanha & 26 \\
\hline 5 & Alemanha & 24 \\
\hline 6 & Canadá & 10 \\
\hline 7 & Suécia & 10 \\
\hline 8 & Suíça & 10 \\
\hline 9 & Brasil & 8 \\
\hline 10 & Itália & 8 \\
\hline 11 & Austrália & 7 \\
\hline 12 & Singapura & 7 \\
\hline 13 & Finlândia & 6 \\
\hline 14 & Japão & 6 \\
\hline 15 & Malásia & 5 \\
\hline 16 & Indonésia & 4 \\
\hline 17 & Irã & 4 \\
\hline 18 & Polônia & 4 \\
\hline 19 & Dinamarca & 3 \\
\hline 20 & França & 3 \\
\hline
\end{tabular}

Fonte: elaboração dos autores.
Na Figura 7 estão os anos mais citados pelos autores. Por exemplo, nos 248 artigos sobre SCRM selecionados existem 212 citações de trabalhos publicados em 2004. Essas citações estão relacionadas a trabalhos científicos de diversas áreas do conhecimento, inclusive o próprio SCRM.

Analisando-se em conjunto a Figura 7 e a Tabela 6 pode-se observar que os pesquisadores sobre o tema SCRM estão desenvolvendo as revisões bibliográficas (fundamentação teórica) de seus trabalhos com base em artigos que, em sua maioria, não se referem ao tema SCRM. A título de exemplo, os anos 2004, 2005 e 2006 somam apenas 9 trabalhos publicados sobre SCRM; porém esses anos são os mais citados nas publicações dos pesquisadores, com 611 registros (212 em 2004, 200 em 2005 e 199 em 2006).

No que tange os periódicos mais citados pelos pesquisadores sobre SCRM, destacam-se, nessa ordem, International Journal of Production Economics, Journal of Operations Management e Supply Chain Management: An International Journal, com mais de 100 citações cada. Levando-se em consideração apenas a Tabela 7, para cada 5 citações que envolvem trabalhos sobre SCRM, uma refere-se a um desses periódicos. Quando se aborda o número de instâncias, essa relação é maior ainda, ou seja, esses três periódicos, juntos, são responsáveis por um terço de todas as citações em publicações sobre SCRM.

Finalizando essa seção e traçando um paralelo com a seção anterior, infere-se que, dos 20 autores que mais publicaram (vide Tabela 3 ) e dos 20 autores mais citados (vide Tabela 5), apenas 4 deles constam

Tabela 5. Relação dos 20 autores mais citados, ordenados pelo número de registros.

\begin{tabular}{cccc}
\hline Ordenação & Autor & Número de Registros & Número de Instâncias \\
\hline 1 & Christopher, M. & 109 & 204 \\
\hline 2 & Tang, C.S. & 96 & 127 \\
\hline 3 & Chopra, S. & 91 & 105 \\
\hline 4 & Zsidisin, A. & 90 & 182 \\
\hline 5 & Juttner, U. & 81 & 118 \\
\hline 6 & Norrman, A. & 74 & 129 \\
\hline 7 & Peck, H. & 74 & 103 \\
\hline 8 & Sheffi, Y. & 71 & 89 \\
\hline 9 & Wagner, S.M. & 71 & 95 \\
\hline 10 & Hallikas, J. & 67 & 90 \\
\hline 11 & Kleindorfer, P.R. & 67 & 95 \\
\hline 12 & Manuj, I. & 66 & 81 \\
\hline 13 & Hendricks, K.B. & 61 & 64 \\
\hline 14 & Lee, H.L. & 58 & 71 \\
\hline 15 & Tang, C. & 55 & 59 \\
\hline 16 & Blackhurst, J.V. & 52 & 57 \\
\hline 17 & Craighead, C.W. & 52 & 73 \\
\hline 19 & Harland, C. & 52 & 59 \\
\hline
\end{tabular}

Fonte: elaboração dos autores. 


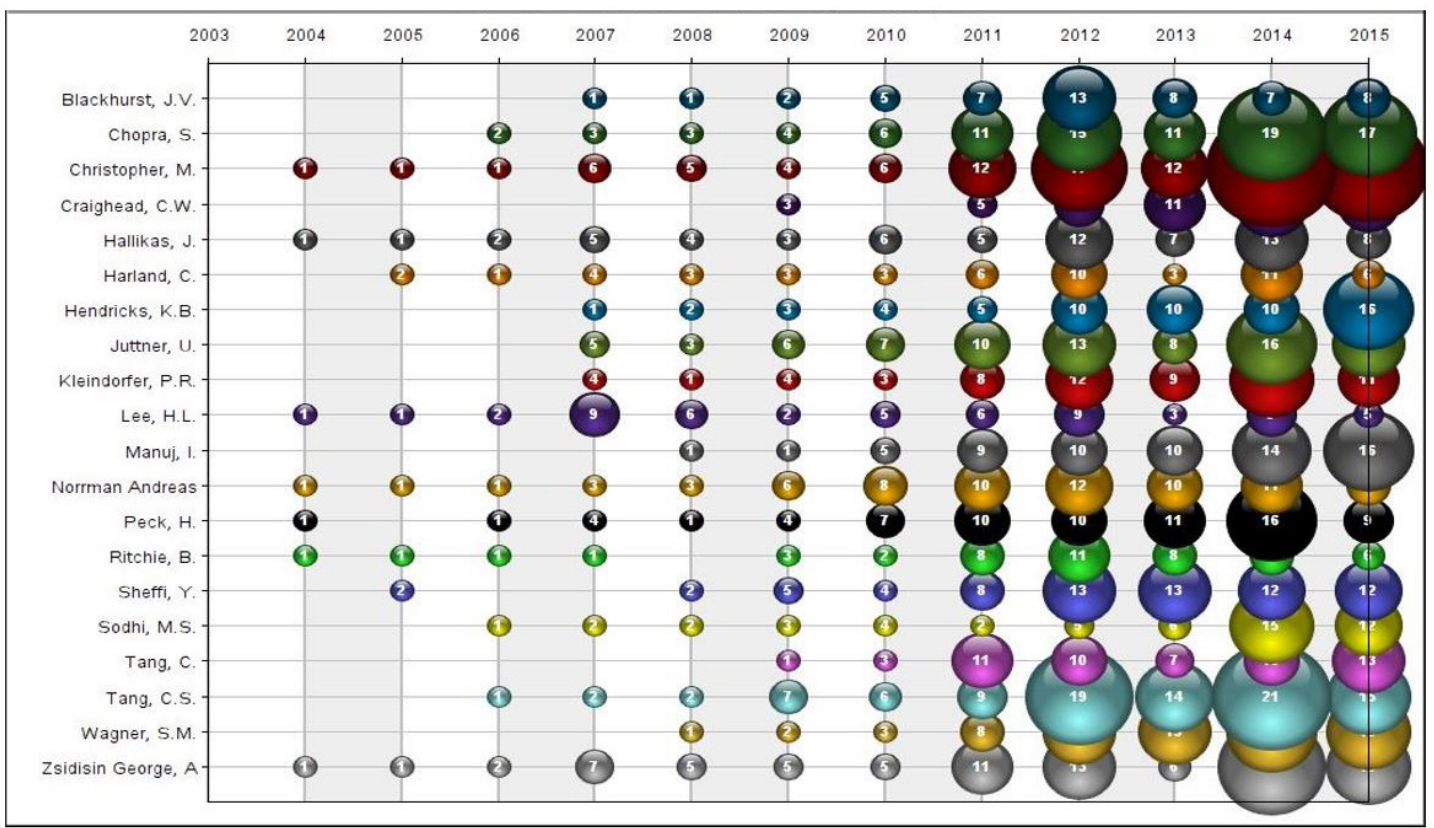

Figura 6. Autores com pesquisa sobre SCRM mais citados até 2015. Fonte: elaboração dos autores a partir do software VantagePoint ${ }^{\circledR}$.

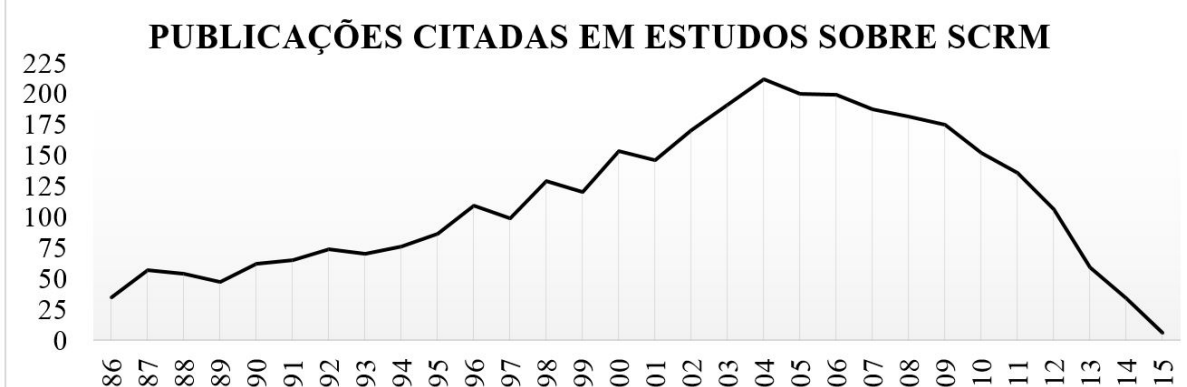

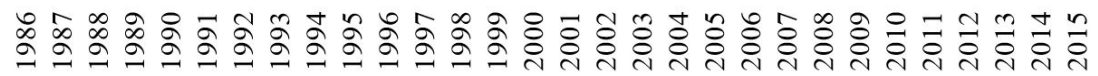

Figura 7. Análise do ano das publicações citadas nas pesquisas sobre SCRM ao longo de 30 anos. Fonte: elaboração dos autores.

Tabela 6. Trabalhos sobre SCRM publicados por ano, nos últimos 12 anos.

\begin{tabular}{ccc}
\hline $\begin{array}{c}\text { Ano de } \\
\text { publicação }\end{array}$ & $\begin{array}{c}\text { Número de } \\
\text { registros }\end{array}$ & $\begin{array}{c}\text { Frequência } \\
\text { acumulada }\end{array}$ \\
\hline 2004 & 2 & 2 \\
\hline 2005 & 3 & 5 \\
\hline 2006 & 4 & 9 \\
\hline 2007 & 15 & 24 \\
\hline 2008 & 12 & 36 \\
\hline 2009 & 12 & 48 \\
\hline 2010 & 14 & 62 \\
\hline 2011 & 26 & 88 \\
\hline 2012 & 36 & 124 \\
\hline 2013 & 38 & 162 \\
\hline 2014 & 51 & 213 \\
\hline 2015 & 35 & 248 \\
\hline
\end{tabular}

Fonte: elaboração dos autores. em ambas as análises. A Figura 8 aborda essa questão, nela, os números no interior de cada coluna em preto indicam os anos que osm artigos de cada autor foram publicados.

Supostamente, vários fatores influenciaram a relação apresentada na Figura 8, tais como o tipo de pesquisa (aplicada ou não), o autor (se antes, durante e após a publicação se tornou reconhecido na área ou não), o ano em que a pesquisa foi publicada (se mais recente ou mais antiga), entre outros aspectos.

\subsection{Subgrupo "Onde pesquisar"}

Aqui foram incluídos os resultados dos periódicos que mais publicaram sobre SCRM e as palavras-chave mais escolhidas pelos autores para representar suas pesquisas. Julgou-se pertinente incluir esses dois 
Tabela 7. Relação dos 20 periódicos mais citados.

\begin{tabular}{clcc}
\hline Ordem & \multicolumn{1}{c}{ Periódicos } & Registros & Instâncias \\
\hline 1 & International Journal of Production Economics & 137 & 501 \\
\hline 2 & Journal of Operations Management & 102 & 324 \\
\hline 3 & Supply Chain Management: An International Journal & 101 & 333 \\
\hline 4 & Management Science & 95 & 224 \\
\hline 5 & International Journal of Physical Distribution \& Logistics Management & 94 & 208 \\
\hline 6 & The International Journal of Logistics Management & 91 & 195 \\
\hline 7 & Production and Operations Management & 88 & 150 \\
\hline 8 & European Journal of Operational Research & 87 & 189 \\
\hline 9 & International Journal of Production Research & 86 & 210 \\
\hline 10 & Journal of Purchasing \& Supply Management & 78 & 127 \\
\hline 11 & MIT Sloan Management Review & 78 & 115 \\
\hline 12 & International Journal of Logistics Research and Applications & 74 & 120 \\
\hline 13 & International Journal of Operations \& Production Management & 64 & 116 \\
\hline 14 & Journal of Business Logistics & 63 & 144 \\
\hline 15 & Journal of Supply Chain Management & 63 & 106 \\
\hline 16 & Decision Sciences & 59 & 100 \\
\hline 17 & Harvard Business Review & 59 & 100 \\
\hline 18 & International Journal of Physical Distribution \& Logistics Management & 58 \\
\hline 19 & Computers in Industry & 35 & 119 \\
\hline 20 & Academy of Management Review & 33 & 37 \\
\hline Fen & 48
\end{tabular}

Fonte: elaboração dos autores.

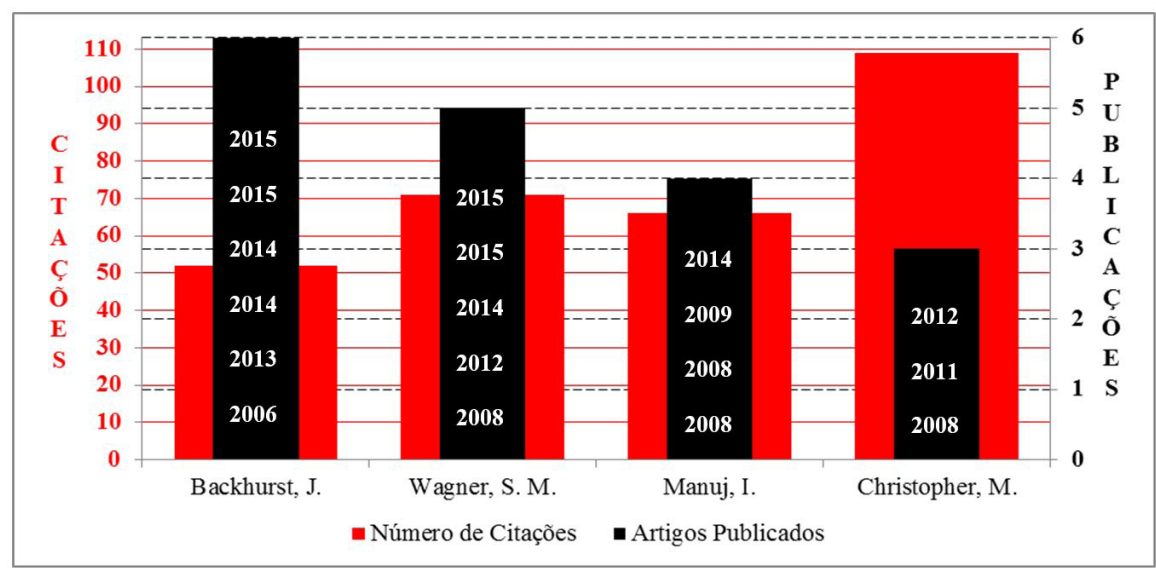

Figura 8. Comparativo entre citação e publicação sobre SCRM de um mesmo autor. Fonte: elaboração dos autores.

estudos aqui, em uma seção exclusiva, por se entender que, quando algum pesquisador tiver interesse em pesquisar sobre SCRM, o fará, principalmente, por palavras-chave ou diretamente nos sítios dos periódicos que mais abordam o assunto. A Tabela 8 organiza as palavras-chave mais selecionadas para representar pesquisas sobre SCRM de 2004 à 2015, tabela essa gerada a partir da mineração de dados pelo software Vantage Point ${ }^{\circledR}$, com base nos 248 artigos que fizeram parte da presente pesquisa.

A Figura 9 complementa a Tabela 8 relacionando, ano a ano, as palavras-chave mais utilizadas. Observa-se na Figura 9 que as primeiras palavras-chave utilizadas para representar pesquisas sobre esse tema foram
"Risk Management" e "Supply Chain Management". Somente após 2012 as palavras-chave "Supply Chain Risk Management" se tornaram representativas, em termos de números absolutos, para pesquisas sobre SCRM.

A Figura 10 ilustra a evolução das três palavras-chave mais utilizadas, tanto em frequência simples como acumulada.

Quanto aos periódicos que mais publicaram sobre SCRM, destacaram-se International Journal of Production Economics, International Journal of Production Research e Supply Chain Management: An International Journal. A Tabela 9 mostra os resultados dessa análise. 
Tabela 8. Relação das 20 palavras-chave mais utilizadas.

\begin{tabular}{clc}
\hline Ordenação & \multicolumn{1}{c}{ Palavras-chave } & Número de registros \\
\hline 1 & "Supply chain risk management" & 113 \\
\hline 2 & "Risk management" & 100 \\
\hline 3 & "Supply chain management" & 62 \\
\hline 4 & "Supply chain" & 49 \\
\hline 5 & "Supply chain risk" & 25 \\
\hline 6 & "Supply chain risk management (SCRM)" & 12 \\
\hline 7 & "Simulation" & 10 \\
\hline 8 & "Risk analysis" & 9 \\
\hline 9 & "Risk assessment" & 9 \\
\hline 10 & "Supply chain disruption" & 9 \\
\hline 11 & "Case study" & 8 \\
\hline 12 & "Resilient supply chain" & 8 \\
\hline 13 & "Risk" & 7 \\
\hline 14 & "Risk identification" & 7 \\
\hline 15 & "SCRM" & 7 \\
\hline 16 & "Supplier selection" & 6 \\
\hline 17 & "Decision making" & 6 \\
\hline 18 & "Uncertainty" & 5 \\
\hline 19 & "Chains" & 5 \\
\hline 20 & "Conceptual framework" & \\
\hline
\end{tabular}

Fonte: elaboração dos autores.

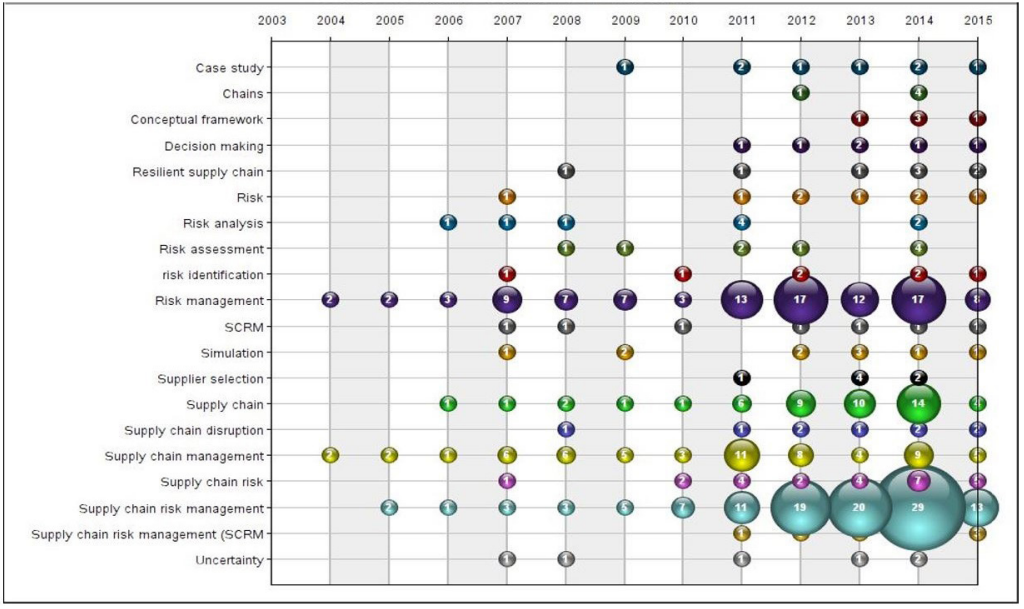

Figura 9. As 20 palavras-chave mais utilizadas em pesquisas sobe SCRM, por ano de publicação. Fonte: elaboração dos autores a partir do software VantagePoint ${ }^{\circledR}$.

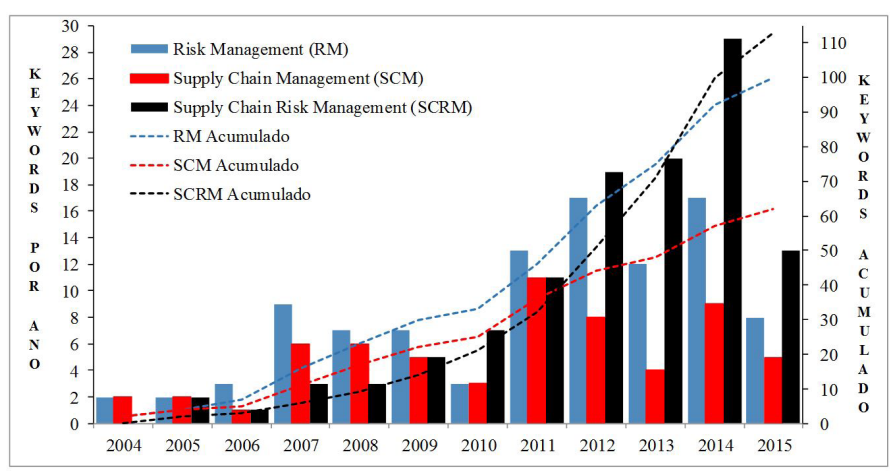

Figura 10. Evolução do uso das três palavras-chave mais utilizadas para representar pesquisas sobre SCRM. Fonte: elaboração dos autores. 
Tabela 9. Relação dos 20 periódicos que mais publicaram sobre SCRM.

\begin{tabular}{clc}
\hline \multirow{2}{*}{ Ordenação } & \multicolumn{1}{c}{ Periódicos } & $\begin{array}{c}\text { Número de } \\
\text { registros }\end{array}$ \\
\hline 1 & International Journal of Production Economics & 14 \\
\hline 2 & International Journal of Production Research & 14 \\
\hline 3 & Supply Chain Management: An International Journal & 12 \\
\hline 4 & CrossTalk Magazine & 9 \\
\hline 5 & The International Journal of Logistics Management & 9 \\
\hline 6 & International Journal of Physical Distribution \& Logistics Management & 9 \\
\hline 7 & International Journal of Logistics Research and Applications & 5 \\
\hline 8 & Journal of Risk Research & 5 \\
\hline 9 & Betriebswirtschaftliche Forschung und Praxis & 3 \\
\hline 10 & Business Process Management Journal & 3 \\
\hline 11 & Computers \& Industrial Engineering & 3 \\
\hline 12 & Computers \& Operations Research & 3 \\
\hline 13 & ICIC Express Letters & 3 \\
\hline 14 & Industrial Management \& Data Systems & 3 \\
\hline 15 & International Journal of Operations \& Production Management & 3 \\
\hline 16 & International Journal of Information Systems and Supply Chain Management & 3 \\
\hline 17 & International Journal of Integrated Supply Management & 3 \\
\hline 18 & International Journal of Retail and Distribution Management & 3 \\
\hline 19 & International Journal of Services and Operations Management & 3 \\
\hline 20 & Journal of Business Logistics & \\
\hline
\end{tabular}

Fonte: elaboração dos autores.

\section{Perspectivas de avanços em pesquisas sobre SCRM}

Com a finalidade de analisar as perspectivas de pesquisas futuras sobre SCRM, esta seção foi subdividida em quatro partes. A primeira subseção (4.1) analisa as principais pesquisas realizadas no período 2004-2015; a segunda subseção (4.2) analisa os principais modelos de SCRM; na terceira subseção (4.3), esses modelos são analisados para posteriores sugestões de melhorias; a última subseção (4.4) organiza as sugestões de alguns pesquisadores sobre o tema SCRM para a realização de pesquisas futuras.

\subsection{Principais pesquisas realizadas sobre SCRM}

Nesta seção, os artigos analisados foram separados em duas fases para que, dessa forma, fosse possível analisar o comportamento do tema ao longo do tempo. Para tanto, os anos foram assim organizados: a primeira fase corresponde ao período 2004-2009 e a segunda, ao período 2010-2015. Depois de separados os artigos, com base em informações fornecidas pelas bases de dados, aplicou-se um diagrama de Pareto em cada uma das fases, visando identificar os $20 \%$ dos artigos mais citados de cada período, de tal forma que os artigos selecionados fossem representativos e os resultados do estudo não ficassem demasiadamente extensos.
Percebe-se que no primeiro período abordado (2004-2009) o conceito gerenciamento de riscos ainda está sendo desenvolvido, tanto pelo baixo número de publicações quanto pelo conteúdo dos artigos, que em sua maioria tratam da criação de modelos de gerenciamento de riscos para as empresas. Um exemplo que sintetiza esse período é o artigo de Finch (2004), no qual o autor analisa a literatura de diversas áreas do conhecimento para então desenvolver um modelo de gerenciamento de riscos, apontando a falta de literatura específica nesse assunto.

Os artigos mais citados do primeiro período (ordenados de forma decrescente pelo número de citações), bem como o que esses trabalhos abordam, se encontram na Tabela 10.

Já no segundo período (2010-2015 - vide Tabela 11), percebe-se uma alteração nas propostas dos trabalhos mais citados, pois o conceito de SCRM já está mais consolidado, permitindo assim aos autores desenvolverem pesquisas mais específicas sobre gerenciamento de riscos, e não mais pesquisas multidisciplinares. Outro fato que pode ser observado é que o tema SCRM não foi estudado apenas seguindo uma metodologia, mas sim explorado tanto em estudos qualitativos quanto quantitativos, confirmando ainda mais a complexidade e abrangência do tema. Por fim, fazendo um paralelo com o primeiro período, ao invés de realizar uma revisão de literatura multidisciplinar, os autores do segundo período já possuem material e maturidade acadêmica sobre o tema para realizarem 
Tabela 10. Os 20\% dos artigos mais citados no período 2004-2009.

\begin{tabular}{ll}
\hline \multicolumn{1}{c}{ Artigo } & \multicolumn{1}{c}{ Pesquisa desenvolvida } \\
\hline Manuj \& Mentzer (2008a) & $\begin{array}{l}\text { Explora o impacto do gerenciamento de riscos e da aplicação de suas } \\
\text { estratégias às cadeias de suprimentos globais. }\end{array}$ \\
\hline Manuj \& Mentzer (2008b) & $\begin{array}{l}\text { Desenvolve um modelo de gerenciamento de riscos em cadeias de suprimentos } \\
\text { globais a partir da integração da literatura de diversas disciplinas. }\end{array}$ \\
\hline Wu \& Olson (2008) & $\begin{array}{l}\text { Compara os resultados simulados de três modelos diferentes de avaliação e } \\
\text { gerenciamento de riscos em cadeias de suprimentos. }\end{array}$ \\
\hline Goh et al. (2007) & $\begin{array}{l}\text { Propõe um modelo para auxiliar cadeias de suprimentos a, entre outras coisas, } \\
\text { gerenciar seus riscos. }\end{array}$ \\
\hline Wu et al. (2006) & $\begin{array}{l}\text { Desenvolve uma nova metodologia para classificação, gerenciamento e } \\
\text { aceitação dos riscos nas cadeias de suprimentos. }\end{array}$ \\
\hline Trkman \& McCormack (2009) & $\begin{array}{l}\text { Apresenta uma nova abordagem para identificação dos riscos, baseada nos } \\
\text { atributos e performance dos fornecedores. }\end{array}$ \\
\hline Ritchie \& Brindley (2007) & $\begin{array}{l}\text { Através da utilização de dois estudos de caso, propõe um quadro de decisões } \\
\text { para auxiliar no gerenciamento de riscos. }\end{array}$ \\
\hline Finch (2004) & $\begin{array}{l}\text { Analisa a literatura para identificar o funcionamento do gerenciamento de riscos } \\
\text { em pequenas e médias empresas. }\end{array}$ \\
\hline Nagali et al. (2008) & $\begin{array}{l}\text { Realiza um estudo de caso para identificar com se dá o gerenciamento de riscos } \\
\text { nas empresas. }\end{array}$ \\
\hline
\end{tabular}

Fonte: elaboração dos autores.

Tabela 11. Os 20\% dos artigos mais citados no período 2010-2015.

\begin{tabular}{|c|c|}
\hline Artigo & Pesquisa desenvolvida \\
\hline Tang \& Musa (2011) & Investiga como está o desenvolvimento das pesquisas em SCRM. \\
\hline Sodhi et al. (2012) & $\begin{array}{l}\text { Realiza um estudo empírico sobre como os fatores operacionais da cadeia de } \\
\text { suprimentos relacionam-se com o gerenciamento de riscos. }\end{array}$ \\
\hline Thun \& Hoenig (2011) & $\begin{array}{l}\text { Estuda o gerenciamento de riscos em cadeias de suprimentos do setor } \\
\text { automotivo. }\end{array}$ \\
\hline Giannakis \& Louis (2011) & $\begin{array}{l}\text { Desenvolve um sistema de decisão multiagente visando mitigação dos riscos nas } \\
\text { cadeias de suprimentos. }\end{array}$ \\
\hline Jüttner \& Maklan (2011) & $\begin{array}{l}\text { Explica a resiliência das cadeias de suprimentos, relacionando esse conceito com } \\
\text { o SCRM. }\end{array}$ \\
\hline Christopher et al. (2011) & $\begin{array}{l}\text { Investiga como os gestores lidam com os riscos provenientes de fontes globais } \\
\text { através de toda a cadeia e que ações são tomadas para mitigá-los. }\end{array}$ \\
\hline Tummala \& Schoenherr (2011) & Propõe uma abordagem para gerenciar riscos nas cadeias de suprimentos. \\
\hline Schmitt \& Singh (2012) & $\begin{array}{l}\text { Demonstra como sistemas resilientes auxiliam na redução dos riscos das cadeias } \\
\text { de suprimentos. }\end{array}$ \\
\hline Schmitt \& Snyder (2012) & $\begin{array}{l}\text { Demonstra a importância da análise a longo prazo dos riscos inerentes às cadeias } \\
\text { de suprimentos. }\end{array}$ \\
\hline Blome \& Schoenherr (2011) & $\begin{array}{l}\text { Analisa estudos de caso a fim de identificar abordagens de sucesso por parte das } \\
\text { empresas no gerenciamento de riscos. }\end{array}$ \\
\hline Lavastre et al. (2012) & $\begin{array}{l}\text { Através de um estudo empírico em empresas francesas, busca descobrir como as } \\
\text { empresas gerenciam os riscos da cadeia de suprimentos em que se inserem. }\end{array}$ \\
\hline Colicchia et al. (2010) & $\begin{array}{l}\text { Apresentam um quadro de decisões simulado que auxilia no gerenciamento de } \\
\text { riscos em escala global. }\end{array}$ \\
\hline Kern et al. (2012) & $\begin{array}{l}\text { Desenvolve, de modo empírico, um modelo de gerenciamento de riscos nas } \\
\text { cadeias de suprimentos. }\end{array}$ \\
\hline Ghadge et al. (2012) & $\begin{array}{l}\text { Analisa o SCRM por um sistema holístico, levando em consideração as diferentes } \\
\text { tipologias envolvidas. }\end{array}$ \\
\hline Olson \& Wu (2011) & Compara o desempenho de ferramentas que auxiliam no gerenciamento de riscos. \\
\hline Wieland \& Wallenburg (2012) & $\begin{array}{l}\text { Testa a hipótese de que o SCRM aumenta a resistência proativamente e diminui o } \\
\text { tempo de reação reativamente. }\end{array}$ \\
\hline Khan et al. (2012) & $\begin{array}{l}\text { Investiga como o alinhamento entre o design do produto e a estratégia da cadeia } \\
\text { de suprimentos impacta na responsividade e resiliência dela. }\end{array}$ \\
\hline
\end{tabular}

Fonte: elaboração dos autores. 
Tabela 11. Continued...

\begin{tabular}{|c|c|}
\hline Artigo & Pesquisa desenvolvida \\
\hline Yang \& Yang (2010) & $\begin{array}{l}\text { Analisa as implicações das medidas mais comuns utilizadas para mitigar os riscos } \\
\text { na cadeia de suprimentos. }\end{array}$ \\
\hline Samvedi et al. (2013) & $\begin{array}{l}\text { Faz uso do método fuzzy AHP para qualificar os riscos inerentes às cadeias de } \\
\text { suprimentos. }\end{array}$ \\
\hline Vilko \& Hallikas (2012) & $\begin{array}{l}\text { Analisa diferentes partes de uma cadeia de suprimentos marítima multimodal } \\
\text { finlandesa para entender como funciona o gerenciamento dos riscos. }\end{array}$ \\
\hline Diabat et al. (2012) & $\begin{array}{l}\text { Cria um modelo que analisa os riscos envolvidos em uma cadeia de suprimentos } \\
\text { do setor alimentício. }\end{array}$ \\
\hline Wu et al. (2013) & $\begin{array}{l}\text { Desenvolve um modelo de programação para gerenciamento de riscos em cadeias } \\
\text { de suprimentos. }\end{array}$ \\
\hline Peng et al. (2014) & $\begin{array}{l}\text { Propõe um modelo que, através de simulações, auxilia no gerenciamento de } \\
\text { riscos das cadeias de suprimentos. }\end{array}$ \\
\hline Grötsch et al. (2013) & $\begin{array}{l}\text { Realiza um estudo empírico para investigar ações proativas de SCRM baseadas } \\
\text { na teoria da contingência. }\end{array}$ \\
\hline Hofmann et al. (2014) & $\begin{array}{l}\text { Realiza uma abordagem multidisciplinar para entender do que se trata e como } \\
\text { funciona o gerenciamento de riscos relacionados à sustentabilidade. }\end{array}$ \\
\hline Chen \& Wu (2013) & $\begin{array}{l}\text { Utiliza o método MFMEA e o método AMP para auxiliar na escolha dos } \\
\text { fornecedores sob a óptica dos riscos da cadeia de suprimentos. }\end{array}$ \\
\hline Wagner \& Neshat (2012) & $\begin{array}{l}\text { Realiza um estudo empírico para medir e comparar as vulnerabilidades das } \\
\text { empresas de diversas categorias. }\end{array}$ \\
\hline Sawik (2011a) & $\begin{array}{l}\text { Desenvolve um modelo de programação para auxiliar na seleção de fornecedores } \\
\text { com menores riscos associados. }\end{array}$ \\
\hline Olson \& Wu (2011) & Realiza uma revisão das publicações sobre SCRM. \\
\hline Tang et al. (2012) & $\begin{array}{l}\text { Investiga o funcionamento do gerenciamento de riscos em um sistema com } \\
\text { variáveis determinadas. }\end{array}$ \\
\hline Thun et al. (2011) & $\begin{array}{l}\text { Analisa o SCRM em empresas de pequeno e médio porte na Alemanha, mais } \\
\text { especificamente do setor automotivo. }\end{array}$ \\
\hline Jia \& Rutherford (2010) & Insere o contexto cultural e regional no SCRM. \\
\hline Govindan et al. (2014) & $\begin{array}{l}\text { Relaciona o gerenciamento verde com o gerenciamento de riscos da cadeia de } \\
\text { suprimentos. }\end{array}$ \\
\hline Sawik (2011b) & $\begin{array}{l}\text { Utiliza os métodos VaR e cVaR para auxiliar na mitigação dos riscos de } \\
\text { fornecimento. }\end{array}$ \\
\hline Lin \& Zhou (2011) & $\begin{array}{l}\text { Investiga o impacto do processo de design dos produtos no gerenciamento de } \\
\text { riscos da cadeia de suprimentos. }\end{array}$ \\
\hline Chae (2015) & $\begin{array}{l}\text { Estuda o impacto que a rede social Twitter tem sobre as práticas das cadeias de } \\
\text { suprimentos, dentre elas o gerenciamento de riscos. }\end{array}$ \\
\hline Dani \& Deep (2010) & $\begin{array}{l}\text { Estudo de caso que analisa o gerenciamento de riscos em uma empresa do setor } \\
\text { alimentício. }\end{array}$ \\
\hline Sun et al. (2012) & Apresenta um modelo de gerenciamento de riscos relacionado aos fornecedores. \\
\hline Ruiz-Torres et al. (2013) & $\begin{array}{l}\text { Constrói um modelo que mitiga riscos através da alocação de demanda ótima } \\
\text { entre um grupo de fornecedores. }\end{array}$ \\
\hline Ghadge et al. (2013) & $\begin{array}{l}\text { Desenvolve um modelo de gerenciamento de riscos e o testa em um estudo de } \\
\text { caso. }\end{array}$ \\
\hline
\end{tabular}

Fonte: elaboração dos autores.

revisões específicas sobre SCRM, como observado em Olson \& Wu (2011).

Os artigos mais citados dessa segunda fase são mostrados na Tabela 11, também em ordem decrescente de citações.

Com base na análise das publicações desses dois períodos, espera-se que no futuro as pesquisas sobre SCRM possam abordar práticas de gerenciamento de riscos com etapas bem definidas, referindo e detalhando explicita e claramente a importância dessas práticas para os gestores, com o intuito de incorporar efetivamente o SCRM como estratégia empresarial, realizando ações proativas com padrões de excelência em sua prática ao longo de toda a cadeia e preparando-se para novas pressões e regulamentações, para além da conformidade legal. Além disso, como a própria evolução histórica coloca em pauta, espera-se que o volume de trabalhos publicados que versem sobre SCRM aumente exponencialmente, considerando-se o cenário competitivo e exigente que se consolida a cada 
dia. Sabe-se, entretanto, que tais fatores dependem da realidade de cada contexto, do avanço tecnológico e da disponibilidade de recursos.

\subsection{Principais modelos de SCRM}

Jüttner et al. (2003) destacaram que eventos como o denominado "bug do milênio", protestos pelos preços de combustíveis, a febre aftosa no Reino Unido e ataques terroristas aos EUA mostraram a vulnerabilidade das modernas cadeias de suprimentos. Os autores revisaram a literatura existente sobre vulnerabilidade em SC e gerenciamento de riscos e os achados foram comparados com as percepções de gerentes de diversas empresas industriais, comerciais e de serviços logísticos envolvidos com as áreas citadas, coletadas por meio de entrevistas.

Buscando estabelecer uma agenda para aprofundamento do assunto e futuras pesquisas, os autores propuseram quatro constructos básicos: as fontes de risco das $\mathrm{SC}$; as consequências dos riscos; os direcionadores dos riscos (como, por exemplo, a globalização, a tendência de terceirização etc.); e as estratégias para mitigação dos riscos, como mostrado na Figura 11.

Gaonkar \& Viswanadham (2007) também propuseram esses mesmos quatro constructos básicos para o gerenciamento de riscos na cadeia de suprimentos. A vulnerabilidade das SC foi também destacada por Norrman \& Jansson (2004): inundação de fábrica da Daimler-Chrysler, incêndio em fábrica de fornecedor da Toyota, queda abrupta da demanda da Cisco; e falhas de planejamento de demanda da Nike foram alguns exemplos apontados pelos autores. Eles apresentam a estrutura e os processos implementados pela Ericsson após um incêndio em um de seus fornecedores que causou severos impactos para a empresa. O modelo utilizado pela empresa é baseado em processos de identificação de riscos; avaliação dos riscos; tratamento dos riscos; e monitoramento dos riscos, tendo, em paralelo, o tratamento de incidentes e planejamento de contingências.

Kleindorfer \& Saad (2005) destacaram, também, diversos exemplos de rupturas, como o terremoto em Taiwan, em 1999, o ataque terrorista às Torres Gêmeas, em 2001, e o apagão na região nordeste do EUA, em 2003. O modelo proposto pelos autores para gerenciamento do risco de ruptura e segurança em redes globais de suprimentos previa o estabelecimento de padrões voluntários para segurança; classificação dos bens e processos em termos de vulnerabilidade; ranking e priorização dos esforços; e iteração para melhoria contínua.

Harland et al. (2003) também apontaram o aumento da complexidade de produtos e serviços, terceirização e globalização como fatores de aumento da complexidade das redes de suprimento, aspectos também defendidos por Singhal et al. (2011). Os autores propuseram uma ferramenta para identificação, avaliação e gerenciamento de riscos, a qual foi testada em quatro estudos de caso da área de Eletrônica. A citada ferramenta, vista na Figura 12, é dividida em seis blocos: mapeamento da rede de suprimentos (estrutura dos atores, métricas e responsabilidades); identificação dos riscos e sua localização (tipo e perdas potenciais); avaliação do risco (probabilidade de ocorrência, estágio no ciclo de vida, exposição, possíveis acionadores e potenciais perdas); gerenciamento do risco (desenvolver posições de risco e desenvolver cenários); formação de estratégia colaborativa de riscos em SC; e implementação de estratégia.

Também destacando a complexidade nas SC, Hallikas et al. (2004), Faisal et al. (2006) e Tuncel \& Alpan (2010) defendem que um processo típico de gerenciamento de riscos em uma empresa é composto de: identificação dos riscos; avaliação dos riscos;

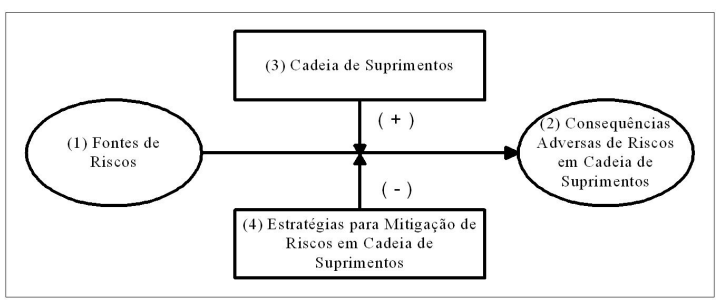

Figura 11. Modelo básico de Gerenciamento de Risco em Cadeia de Suprimentos. Fonte: Jüttner et al. (2003).

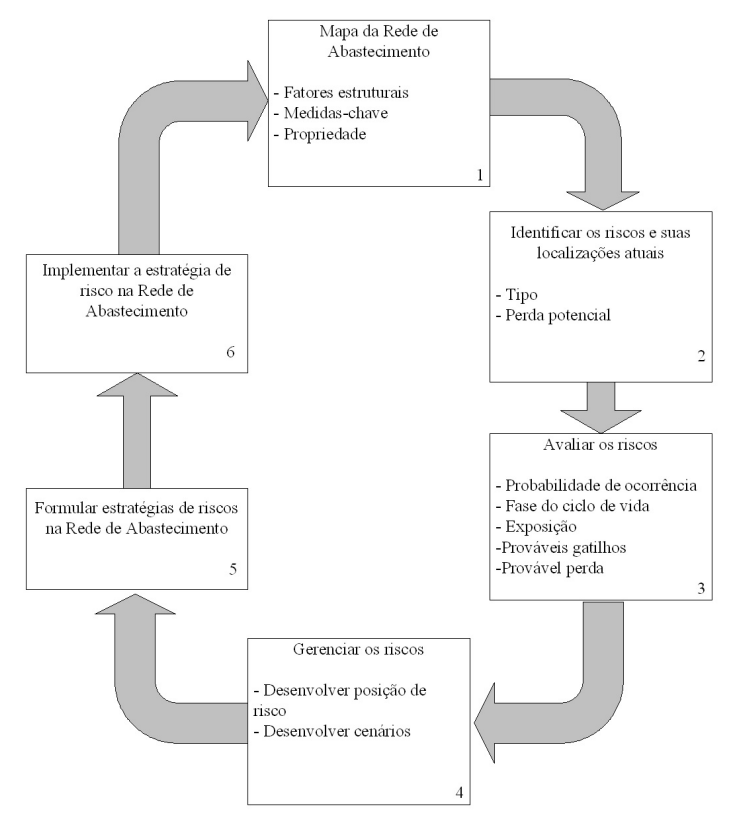

Figura 12. Ferramenta de Riscos em Rede de Abastecimento. Fonte: Harland et al. (2003). 


\begin{tabular}{|c|c|c|c|c|}
\hline $\begin{array}{c}\text { Contexto do } \\
\text { Risco e seus } \\
\text { Condutores } \\
\text { Nível Primário } \\
\text { Nivel } \\
\text { Secundário }\end{array}$ & $\begin{array}{c}\text { Influenciadores do } \\
\text { Gerenciamento de } \\
\text { Riscos } \\
\text { Recompensas } \\
\text { Risco da Cadeia de } \\
\text { Suprimentos } \\
\text { Prazos } \\
\text { Portifólio }\end{array}$ & $\begin{array}{c}\begin{array}{c}\text { Tomador(es) } \\
\text { de Decisão }\end{array} \\
\text { Percepções } \\
\text { Perfil do Risco } \\
\text { Atitudes } \\
\text { Experiências }\end{array}$ & $\begin{array}{c}\text { Respostas do } \\
\text { Gerenciamento } \\
\text { de Riscos } \\
\text { Aceitar o Risco } \\
\text { Evitar } \\
\text { Mitigar } \\
\text { Monitorar }\end{array}$ & $\begin{array}{l}\text { Resultados de } \\
\text { Desempenho } \\
\text { Relacionado ao } \\
\text { Lucro } \\
\text { Posicionamento } \\
\text { Estratégico } \\
\text { Pessoal }\end{array}$ \\
\hline
\end{tabular}

Figura 13. Estrutura do SCRM. Fonte: Ritchie \& Brindley (2007).

decisão e implementação das ações de gerenciamento do risco; e monitoramento dos riscos.

O modelo proposto por Ritchie \& Brindley (2007) apresenta uma abordagem distinta, propondo cinco componentes: contexto dos riscos e seus direcionadores; influenciadores do gerenciamento de riscos (incluindo prazos e portfólio); tomadores de decisão (percepções, perfil, atitudes e experiências); respostas para gerenciamento de riscos (aceitação do risco, evitar, mitigar e monitorar); e desempenho final (relacionado ao perfil, posicionamento estratégico e pessoal), como mostrado na Figura 13.

Já Wu et al. (2006), focando nos riscos da logística de entrada (inbound), buscaram identificar, também por revisão de literatura e entrevistas, os fatores de risco. Propuseram um modelo para o gerenciamento desses riscos, composto por quatro componentes: classificação dos riscos na SC (controláveis internos, parcialmente controláveis internos, internos incontroláveis, externos controláveis, externos parcialmente controláveis e externos incontroláveis); identificação dos riscos nos fornecedores; cálculo dos riscos por meio do método Analytic Hierarchy Planning - AHP (Saaty, 1994); e simulação computacional (O'Kane et al., 2000).

Kern et al. (2012) também focaram na logística de entrada, propondo um modelo para gerenciamento de riscos composto de identificação de riscos; avaliação dos riscos; mitigação dos riscos; e desempenho frente aos riscos, tendo adicionalmente examinado o impacto do processo de melhoria contínua nesses constructos, como mostrado na Figura 14.

Matook et al. (2009) optaram por focar seu estudo no gerenciamento de riscos nos fornecedores. Os autores propuseram um modelo composto por cinco componentes: identificação de riscos nos fornecedores; avaliação de riscos nos fornecedores; reporte e decisão sobre riscos dos fornecedores; respostas de gerenciamento de riscos de fornecedores; e desempenho dos fornecedores quanto ao risco.

Blome \& Schoenherr (2011) também focaram seu estudo no lado dos fornecedores, utilizando múltiplos

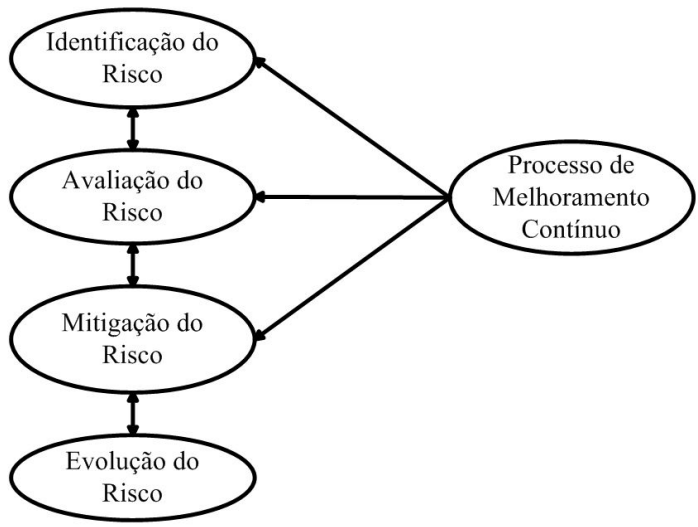

Figura 14. Modelo conceitual de SCRM. Fonte: Kern et al. (2012).

estudos de caso em oito companhias europeias para identificar abordagens e experiências de sucesso, tendo desenvolvido um modelo de gerenciamento de riscos em momentos de crises econômico-financeiras. O modelo proposto continha as seguintes etapas para gerenciamento de riscos na SC e na própria empresa: identificação dos riscos; análise dos riscos; mitigação dos riscos; e monitoramento dos riscos.

A análise em momentos de crises econômicas foi também o foco do estudo de Giannakis \& Louis (2011), que apresentaram um modelo de um sistema multiagente de apoio à decisão para gerenciamento de rupturas e mitigação de riscos em cadeias de suprimento em manufatura. O processo de gerenciamento de riscos seria composto por quatro estágios: identificação dos riscos; avaliação dos riscos; decisão e implementação das ações de gerenciamento do risco; e otimização.

Cohen \& Kunreuther (2007), por outro lado, propuseram um modelo mais detalhado de análise de riscos, composto por: avaliação dos riscos e análise de vulnerabilidade; modelagem dos riscos; estratégias de gerenciamento de riscos; e avaliação das estratégias, como pode ser visto na Figura 15. 
O estudo de Manuj \& Mentzer (2008a) que, ao contrário dos autores anteriores, focou no gerenciamento de riscos nas cadeias globais de suprimento, apontou que o processo para gerenciamento e mitigação de riscos nessa área seria composto por cinco passos: identificação dos riscos (classificados em suprimentos, operações, demanda e segurança); análise e avaliação dos riscos (análise da decisão, estudos de caso e apoio na percepção); seleção do gerenciamento de riscos apropriado (ou seja, a estratégia proposta: evitar, postergar, especular, limitar, controlar, dividir/transferir e segurar); implementação das estratégias (tendo como habilitadores o gerenciamento da complexidade, o aprendizado organizacional, tecnologia da informação e indicadores de desempenho); e mitigação dos riscos (preparando-se para eventos imprevistos), como disposto nas Figuras 16 e 17.

\subsection{Constatações sobre o processo de realização do SCRM e suas etapas}

Analisando-se a bibliografia sobre SCRM, observa-se que a maioria dos pesquisadores sobre o tema defende que esse conhecimento emerge como

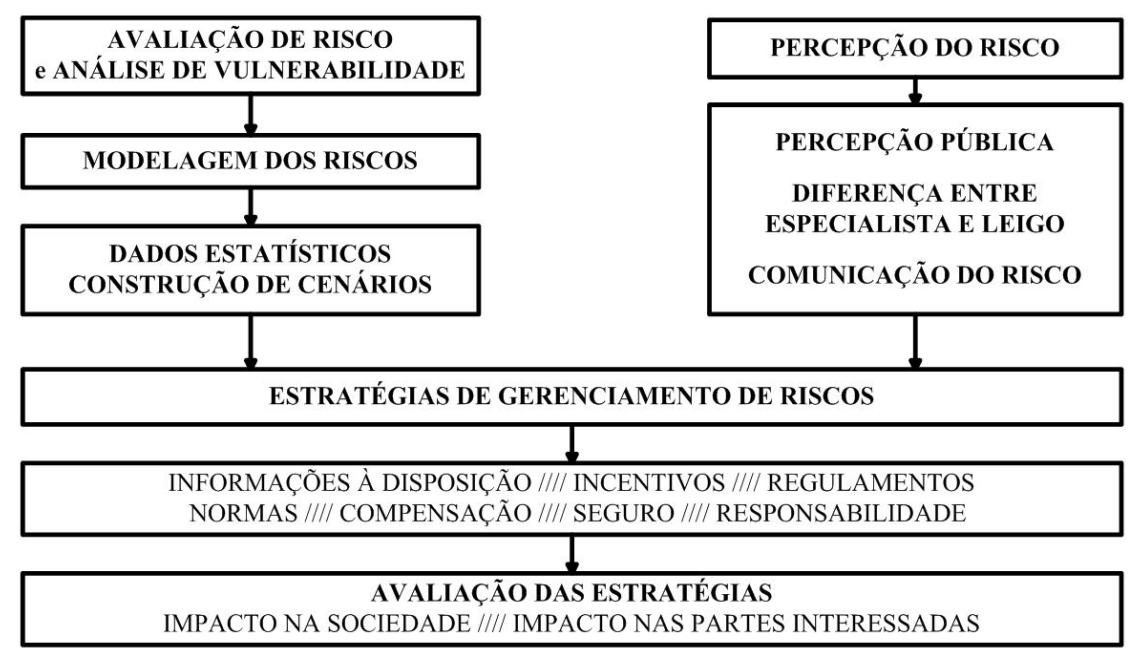

Figura 15. Estrutura Conceitual para Análise de Riscos. Fonte: adaptado de Cohen \& Kunreuther (2007).

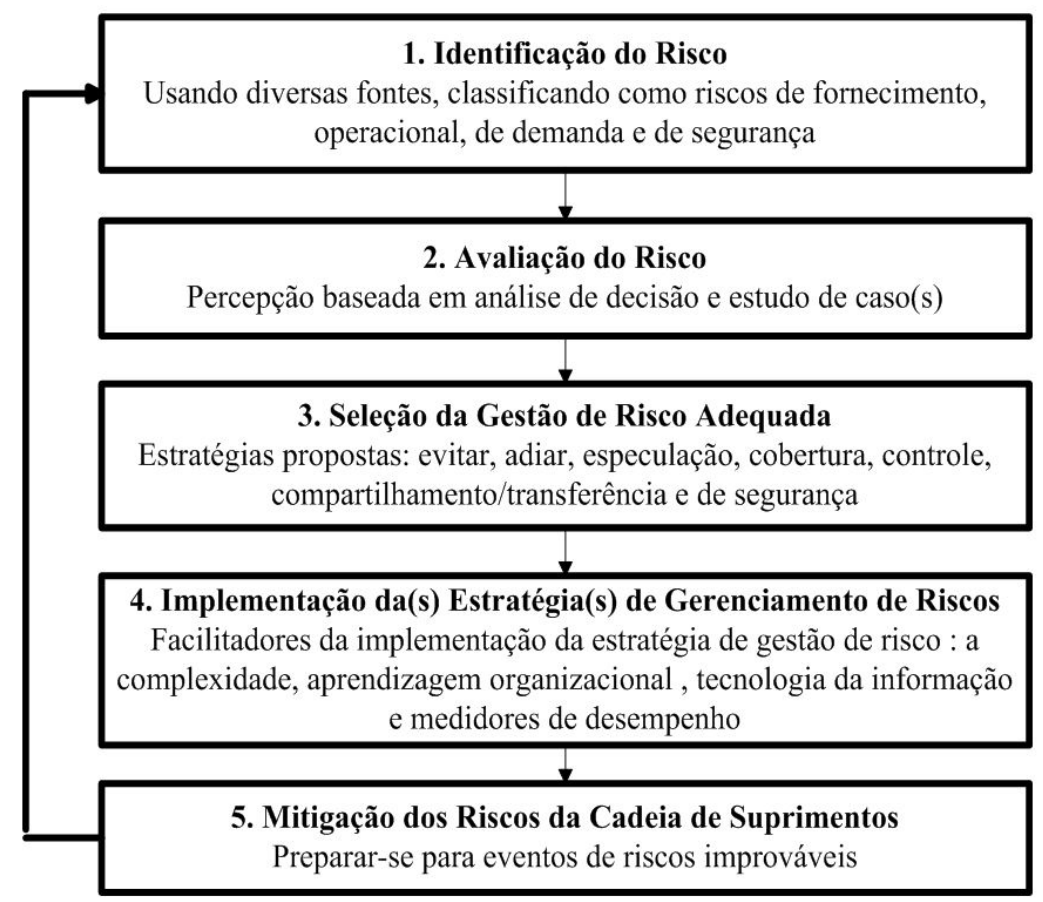

Figura 16. Um processo de 5 etapas para o SCRM. Fonte: Manuj \& Mentzer (2008b). 


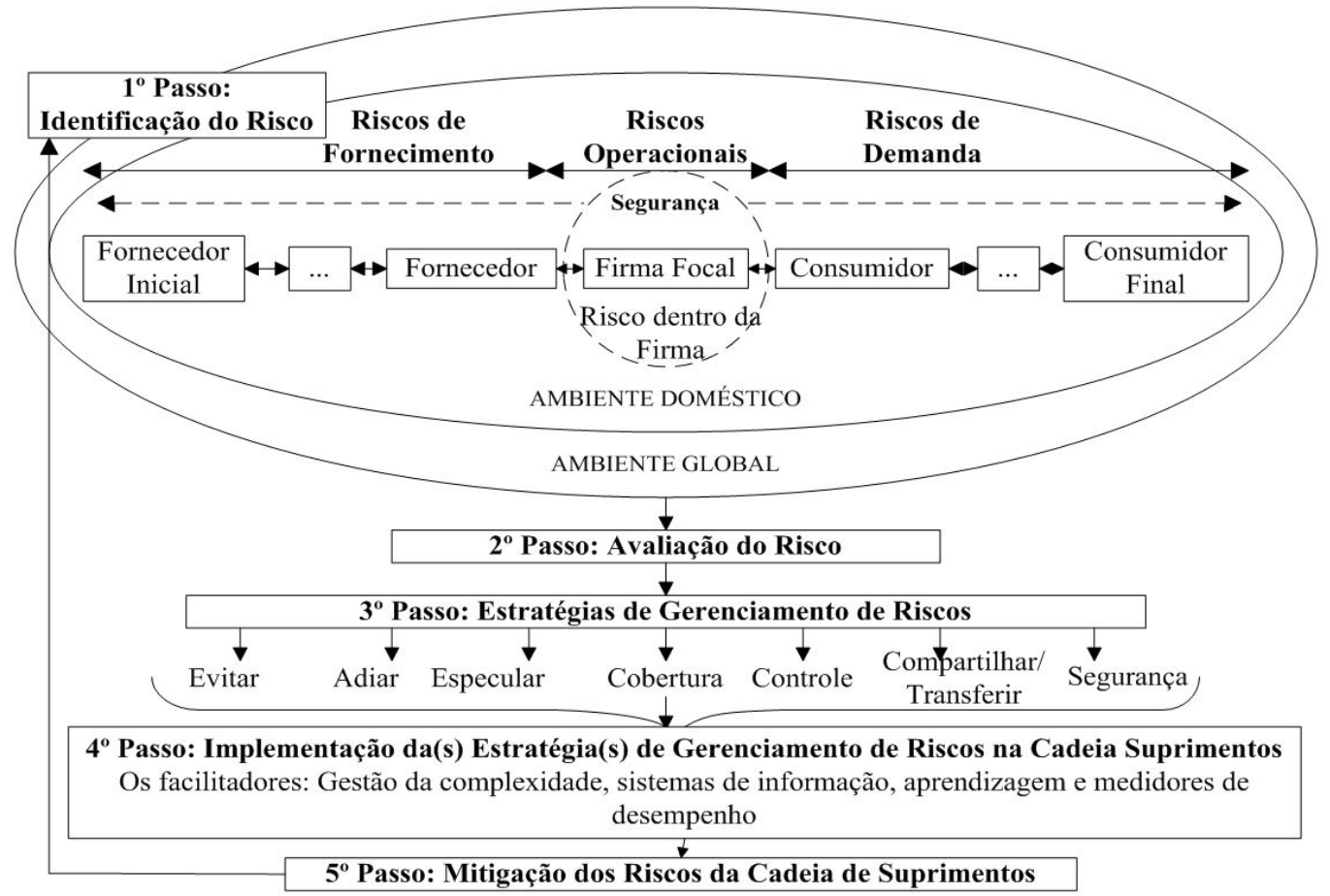

Figura 17. Estrutura do SCRM. Fonte: Manuj \& Mentzer (2008b).

um importante processo no SCM, com o objetivo principal de identificar as fontes potenciais de risco e sugerir medidas adequadas para mitigá-las.

Entretanto, também se observa uma falta de consenso entre esses mesmos pesquisadores quando o assunto diz respeito às etapas que devem ser desenvolvidas no SCRM, tanto em sua quantidade como em suas ações. Somente como exemplo, Wu et al. (2006), Khan \& Burnes (2007), Oehmen et al. (2009) e Singhal et al. (2011) defendem que o SCRM deve ser realidado em um processo mínimo de três etapas; mas se diferenciam em seus procedimentos, de autor para autor.

Outros autores, como Hallikas et al. (2004), Kleindorfer \& Saad (2005), Manuj \& Mentzer (2008a), Tummala \& Schoenherr (2011), defendem procedimentos diferentes, com mais de três etapas. Já Ritchie \& Brindley (2007) defendem a realização de sete etapas para o Gerenciamento de Riscos na Cadeia de Suprimentos. Como resultado de uma síntese nesse assunto, o Quadro 1 ilustra como 23 autores que abordaram as etapas do SCRM em seus trabalhos.

Há de se levar em conta que os problemas em cadeias de suprimentos nem sempre apresentam as mesmas exigências e, portanto, os mesmos métodos de solução. No entanto, ao se analisar o Quadro 1, infere-se que:

- 86,96\% abordam a etapa "identificação de riscos" em suas pesquisas;
- $82,61 \%$ abordam a etapa "avaliação dos riscos" em suas pesquisas;

- 60,87\% abordam a etapa "proposição de estratégias" em suas pesquisas;

- 56,52\% abordam a etapa "mitigação dos riscos" em suas pesquisas.

Diante disso, pode-se questionar: a etapa "controle de riscos" não é relevante para o SCRM? Justifica-se esse questionamento, pois apenas $30,43 \%$ dos pesquisadores abordaram essa etapa em suas pesquisas.

Apesar de não ter sido o foco desta pesquisa analisar a definição intrínseca de cada uma das etapas do Quadro 1, supõe-se que algumas dessas etapas são apenas variantes de um mesmo significado, como, por exemplo "monitorização de riscos" e "controle de riscos". Tal análise não passa de uma suposição, pois dos 23 trabalhos, 3 refutam essa observação por considerarem essas etapas como complementares (Hallikas et al., 2004; Ritchie \& Brindley, 2007; Tummala \& Schoenherr, 2011), sendo, portanto, necessários estudos mais aprofundados sobre essa questão.

Outro aspecto importante está em "como" realizar cada uma das etapas de SCRM, ou seja, quais "ferramentas", "técnicas", "abordagens" e "procedimentos" usar, por exemplo, para "identificar", "avaliar", "mitigar" e "monitorar" os riscos em cadeias de suprimentos. $\mathrm{Na}$ análise dos 248 artigos que fizeram parte deste 
Quadro 1. Etapas sugeridas para o SCRM por diversos autores que pesquisaram sobre o tema.

\begin{tabular}{|c|c|c|c|c|c|c|c|c|c|c|c|c|c|c|}
\hline \multirow[b]{2}{*}{ 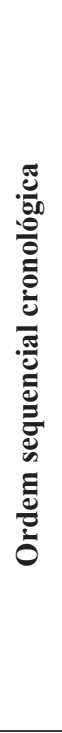 } & \multirow[b]{2}{*}{ Pesquisadores sobre SCRM } & \multicolumn{13}{|c|}{ Relação das etapas para a realização do SCRM } \\
\hline & & 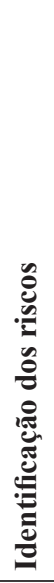 & 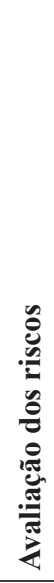 & 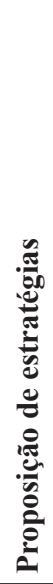 & 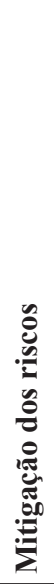 & 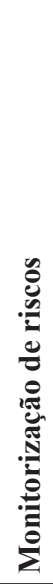 & 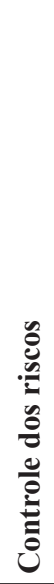 & 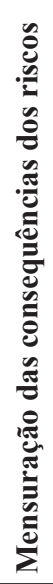 & 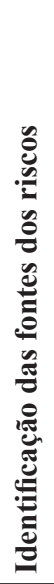 & 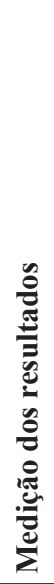 & 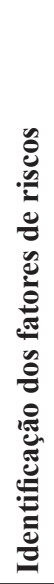 & 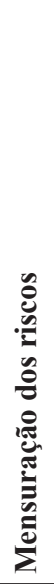 & 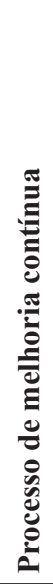 & 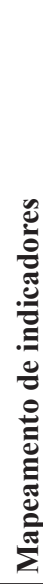 \\
\hline 1 & Jüttner et al. (2003) & & $\mathrm{X}$ & & $\mathrm{X}$ & $\mathrm{X}$ & & & $\mathrm{X}$ & & & & & \\
\hline 2 & Harland et al. (2003) & $\mathrm{X}$ & $\mathrm{X}$ & $\mathrm{X}$ & & & & $\mathrm{X}$ & & & & & & $\mathrm{X}$ \\
\hline 3 & Hallikas et al. (2004) & $\mathrm{X}$ & $\mathrm{X}$ & & $\mathrm{X}$ & $\mathrm{X}$ & $\mathrm{X}$ & & & & & & & \\
\hline 4 & Norrman \& Jansson (2004) & $\mathrm{X}$ & $\mathrm{X}$ & $\mathrm{X}$ & & $\mathrm{X}$ & & & & & & & & \\
\hline 5 & Kleindorfer \& Saad (2005) & & $\mathrm{X}$ & $\mathrm{X}$ & $\mathrm{X}$ & & & & $\mathrm{X}$ & & & & & \\
\hline 6 & Zsidisin et al. (2005) & $\mathrm{X}$ & $\mathrm{X}$ & $\mathrm{X}$ & & & $\mathrm{X}$ & & & & & & & \\
\hline 7 & Wu et al. (2006) & $\mathrm{X}$ & & & $\mathrm{X}$ & & & & & & & $\mathrm{X}$ & & \\
\hline 8 & Faisal et al. (2006) & $\mathrm{X}$ & $\mathrm{X}$ & & $\mathrm{X}$ & $\mathrm{X}$ & & & & & & & & \\
\hline 9 & Khan \& Burnes (2007) & $\mathrm{X}$ & & $\mathrm{X}$ & & & & $\mathrm{X}$ & & & & & & \\
\hline 10 & Ritchie \& Brindley (2007) & $\mathrm{X}$ & & $\mathrm{X}$ & & $\mathrm{X}$ & $\mathrm{X}$ & & $\mathrm{X}$ & $\mathrm{X}$ & $\mathrm{X}$ & & & \\
\hline 11 & Gaonkar \& Viswanadham (2007) & & $\mathrm{X}$ & $\mathrm{X}$ & & & & $\mathrm{X}$ & $\mathrm{X}$ & & $\mathrm{X}$ & & & \\
\hline 12 & Cohen \& Kunreuther (2007) & $\mathrm{X}$ & $\mathrm{X}$ & $\mathrm{X}$ & $\mathrm{X}$ & & & $\mathrm{X}$ & & $\mathrm{X}$ & & & & \\
\hline 13 & Manuj \& Mentzer (2008a) & $\mathrm{X}$ & $\mathrm{X}$ & $\mathrm{X}$ & $\mathrm{X}$ & & & $\mathrm{X}$ & & & & & & \\
\hline 14 & Matook et al. (2009) & $\mathrm{X}$ & & $\mathrm{X}$ & & & $\mathrm{X}$ & & & $\mathrm{X}$ & & & & \\
\hline 15 & Oehmen et al. (2009) & $\mathrm{X}$ & $\mathrm{X}$ & & $\mathrm{X}$ & & & & & & & & & \\
\hline 16 & Tuncel \& Alpan (2010) & $\mathrm{X}$ & $\mathrm{X}$ & $\mathrm{X}$ & & $\mathrm{X}$ & & & & & & & & \\
\hline 17 & Giannakis \& Louis (2011) & $\mathrm{X}$ & $\mathrm{X}$ & $\mathrm{X}$ & $\mathrm{X}$ & & $\mathrm{X}$ & & & & & & & \\
\hline 18 & Tummala \& Schoenherr (2011) & $\mathrm{X}$ & $\mathrm{X}$ & & $\mathrm{X}$ & $\mathrm{X}$ & $\mathrm{X}$ & & & & & $\mathrm{X}$ & & \\
\hline 19 & Blome \& Schoenherr (2011) & $\mathrm{X}$ & $\mathrm{X}$ & & $\mathrm{X}$ & & $\mathrm{X}$ & & & & & & & \\
\hline 20 & Guo (2011) & $\mathrm{X}$ & $\mathrm{X}$ & $\mathrm{X}$ & & $\mathrm{X}$ & & & & & & & & \\
\hline 21 & Singhal et al. (2011) & $\mathrm{X}$ & $\mathrm{X}$ & & $\mathrm{X}$ & & & & & & & & & \\
\hline 22 & Kern et al. (2012) & $\mathrm{X}$ & $\mathrm{X}$ & & $\mathrm{X}$ & & & & & $\mathrm{X}$ & & & $\mathrm{X}$ & \\
\hline 23 & Rangel et al. (2015) & $\mathrm{X}$ & $\mathrm{X}$ & $\mathrm{X}$ & & $\mathrm{X}$ & & & & & & & & \\
\hline & TOTAL POR ETAPA & 20 & 19 & 14 & 13 & 09 & 07 & 06 & 04 & 04 & 02 & 02 & 01 & 01 \\
\hline
\end{tabular}

Fonte: elaboração dos autores.

estudo, observou-se que a maioria das pesquisas não "ensina" o leitor, o profissional, ou outro pesquisador "como" realizar o SCRM.

\subsection{Recomendações para futuras pesquisas em SCRM}

Esta etapa organizou, a partir dos 248 artigos pesquisados, várias recomendações para pesquisas futuras, conforme exposto na Tabela 12. Essa tabela tem por finalidade direcionar potenciais pesquisadores sobre tópicos que não foram estudados (gaps) pelos autores pesquisados, mas que foram citados como importantes nos respectivos artigos.

Como delimitador dos resultados da Tabela 12, ressalve-se que não foi analisado se alguma das recomendações virou tema de pesquisa de alguma outra publicação, ou seja, provavelmente algumas dessas recomendações já tenham sido estudadas ou implementadas. 
Tabela 12. Recomendações de pesquisas futuras sobre SCRM, em ordem cronológica.

\begin{tabular}{|c|c|}
\hline Artigo & Recomendações \\
\hline Norrman \& Jansson (2004) & $\begin{array}{l}\text { Estudar a relação entre o gerenciamento de riscos em cadeias de suprimentos e os } \\
\text { princípios do gerenciamento logístico da cadeia de suprimentos. }\end{array}$ \\
\hline Wu et al. (2006) & Explorar o método em mais empresas, utilizando também dados históricos. \\
\hline Faisal et al. (2006) & Avaliar o modelo proposto sob uma óptica estatística. \\
\hline Goh et al. (2007) & Aplicar o modelo em uma cadeia de suprimentos em larga escala. \\
\hline Ritchie \& Brindley (2007) & $\begin{array}{l}\text { Se concentrar menos na exploração dos componentes do quadro proposto e mais } \\
\text { na dinâmica de interação entre eles. }\end{array}$ \\
\hline Autry \& Bobbitt (2008) & Continuar os estudos sobre SCSO (Supply Chain Security Orientation). \\
\hline Manuj \& Mentzer (2008a) & $\begin{array}{l}\text { Refinar e testar o modelo proposto através de dados quantitativos e qualitativos } \\
\text { acerca de diferentes indústrias. }\end{array}$ \\
\hline Manuj \& Mentzer (2008b) & $\begin{array}{l}\text { Identificar como o modelo proposto no artigo está relacionado com a performance } \\
\text { das cadeias de suprimento. }\end{array}$ \\
\hline Williams et al. (2008) & $\begin{array}{l}\text { Desenvolver as pesquisas sugeridas no artigo sobre estratégias de gerenciamento } \\
\text { de riscos. }\end{array}$ \\
\hline Ojha \& Gokhale (2009) & Avaliar o impacto financeiro e operacional do modelo proposto no artigo. \\
\hline Manuj et al. (2009) & Usar outras ferramentas de simulação e outras abordagens para o estudo proposto. \\
\hline Trkman \& McCormack (2009) & $\begin{array}{l}\text { Fazer um artigo com múltiplos estudos de caso envolvendo não só os fornecedores } \\
\text { da firma focal, mas também os fornecedores dos fornecedores. }\end{array}$ \\
\hline Sodhi \& Tang (2009) & $\begin{array}{l}\text { Usar a análise de Monte Carlo como outra abordagem para o modelo apresentado } \\
\text { no artigo. }\end{array}$ \\
\hline Pujawan \& Geraldin (2009) & Levar em consideração a interdependência entre as variáveis do estudo. \\
\hline $\begin{array}{l}\text { Moeinzadeh \& Hajfathaliha } \\
(2009)\end{array}$ & $\begin{array}{l}\text { Realizar o estudo desse artigo usando outros métodos e técnicas; explorar mais } \\
\text { casos e conduzir mais estudos empíricos acerca do modelo proposto. }\end{array}$ \\
\hline Jüttner \& Maklan (2011) & Utilizar mais estudos de caso; abordar o tema por outras perspectivas. \\
\hline Olson \& Wu (2011) & $\begin{array}{l}\text { Utilizar dados reais de uma organização específica a fim de testar o modelo na } \\
\text { prática. }\end{array}$ \\
\hline
\end{tabular}

Tummala \& Schoenherr (2011) Investigar como as informações do sistema interno das empresas podem ser usadas para alimentar o modelo exposto no artigo.

Giannakis \& Louis (2011) $\quad$ Realizar simulações para avaliar a performance do quadro proposto.

Sawik (2011a) Utilizar diferentes abordagens para o modelo proposto.

Thun \& Hoenig (2011) Utilizar o mesmo método em indústrias de outros setores, inclusive com uma pesquisa internacional.

Blome \& Schoenherr (2011) Replicar o estudo em outras indústrias, em outros países; realizar uma pesquisa

Blome \& Schoenherr (2011) internacional em larga escala.

Sawik (2011b) Aumentar o tamanho da amostra e de variáveis utilizadas na aplicação do modelo.

Tse et al. (2011) Testar empiricamente o modelo proposto no artigo.

Sodhi et al. (2012) Desenvolver o mesmo estudo em outras empresas de diferentes contextos.

Lockamy \& Mccormack Examinar perfis de riscos de fornecimento e redes de fornecimento pelo método (2012) Rede Bayesiana.

Kern et al. (2012) Replicar o estudo em escala global, coletando informações acerca das empresas participantes de várias fontes. Conduzir estudos de casos similares a fim de comparar o nível de alinhamento da

Khan et al. (2012) cadeia de suprimentos, analisando essa informação sob a óptica do gerenciamento de riscos.

Wieland \& Wallenburg (2012) Testar a proposta do estudo empiricamente; analisar indústrias de outros países.

Schmitt \& Snyder (2012) Desenvolver um estudo de caso comparando dois fornecedores: um deles utilizando a metodologia proposta pelo artigo e o outro não.

Schmitt \& Singh (2012) Aplicar o estudo em outros tipos de indústrias.

Tang et al. (2012) Investigar a política de preços de uma empresa e sua influência em um sistema contendo dois produtos substitutos.

Kumar \& Harrison (2012) Aplicar o modelo proposto de forma mais profunda e auxiliar as empresas que desejam investir em estratégias para um plano de contingência de rompimentos.

Fonte: elaboração dos autores 
Tabela 12. Continuação...

\begin{tabular}{ll}
\hline \multicolumn{1}{c}{ Artigo } & \multicolumn{1}{c}{ Recomendações } \\
\hline Vilko \& Hallikas (2012) & $\begin{array}{l}\text { Recolher mais dados das empresas e envolver outras localizações geográficas; } \\
\text { analisar fatores qualitativos e financeiros dos riscos e seus efeitos no contexto da } \\
\text { cadeia de suprimentos. }\end{array}$ \\
\hline Wever et al. (2012) & Levar em consideração a estrutura da cadeia de suprimentos em conta. \\
\hline Liew \& Lee (2012) & $\begin{array}{l}\text { Incluir outras matérias-primas na análise proposta pelo artigo; estudar a eficiência } \\
\text { do gerenciamento de riscos na mitigação do risco de flutuação de preço das } \\
\text { matérias-primas. }\end{array}$ \\
\hline Aloini et al. (2012) & $\begin{array}{l}\text { Realizar estudos de caso para comprovar as descobertas da análise de literatura } \\
\text { em questão. }\end{array}$ \\
\hline Son \& Orchard (2013) & $\begin{array}{l}\text { Realizar estudos comparativos em que apenas algumas empresas são expostas ao } \\
\text { modelo proposto e outras não. }\end{array}$ \\
\hline Ghadge et al. (2013) & Aplicar o modelo em mais empresas ao invés de apenas uma. \\
\hline Ekwall \& Lantz (2013) & Utilizar outras fontes de dados e desenvolver uma abordagem multidisciplinar. \\
\hline Golgeci \& Ponomarov (2013) & $\begin{array}{l}\text { Desenvolver novos estudos relacionando o conceito de inovação com } \\
\text { gerenciamento de riscos. }\end{array}$ \\
\hline
\end{tabular}

Ruiz-Torres et al. (2013)

Analisar cadeias de suprimentos mais complexas e considerar outras variáveis na análise dos fornecedores.

Wu et al. (2013) Aumentar o alcance da pesquisa e incluir mais variáveis na análise.

Chen \& Wu (2013)

Dar mais atenção aos critérios de seleção de fornecedores usados pelas empresas; utilizar outros métodos para saber se geram resultados similares aos do artigo.

Urciuoli et al. (2014) Desenvolver novas ferramentas de gerenciamento de riscos; utilizar simulações e abordagens matemáticas além do estudo de caso.

Testar de forma empírica os argumentos apresentados no artigo e realizar estudos

Vilko et al. (2014) qualitativos explorando os vários tipos de processos relacionados à tomada de decisão em diferentes níveis de incerteza.

Liu et al. (2014) Considerar outros tipos de veículos na análise; estudar o mesmo tópico usando diferentes metodologias; fazer a pesquisa usando dados reais.

Cantor et al. (2014b) Avaliar o modelo proposto sob uma óptica estatística e expandir a pesquisa com relação ao número de empresas pesquisadas.

Peng et al. (2014) Coletar a opinião de especialistas através de questionários acerca do planejamento logístico.

Benedek et al. (2014) Continuar a pesquisa abordando outros pontos de vista em diferentes empresas. Focar os estudos nas empresas que conseguem gerenciar com sucesso seus riscos

Hofmann et al. (2014) relacionados ao conceito de sustentabilidade; refinar o propósito da pesquisa incluindo outras variáveis.

Manuj et al. (2014) Desenvolver métodos complementares fora do ambiente simulado; aumentar o número de variáveis consideradas.

Cantor et al. (2014a) Olhar para outras variáveis, aprofundando a pesquisa apresentada no artigo.

Chan et al. (2015) Testar o método proposto em outras cadeias de suprimentos.

Marija et al. (2015) Utilizar o mesmo método a fim de comparar com os resultados do presente artigo.

Davarzani et al. (2015) Realizar estudos empíricos para comprovar a proposta do artigo.

Nooraie \& Parast (2015) Aumentar o número de variáveis contextuais e organizacionais consideradas no artigo.

Mizgier et al. (2015) Aplicar o modelo apresentado em um caso real; considerar um maior número de variáveis.

Li et al. (2015) Investigar outras práticas de identificação de riscos; utilizar outras teorias no mesmo contexto pesquisado.

Chae (2015) Aumentar o número de palavras-chave utilizadas na pesquisa; aumentar o número de pesquisas relacionando as cadeias de suprimentos com as redes sociais.

Rajesh \& Ravi (2015) Aumentar o número de estudos de caso para validar a pesquisa; fazer uso de softwares que auxiliem na construção do modelo proposto.

Yu et al. (2015) Fazer testes empíricos sobre a teoria apresentada.

Kilubi \& Haasis (2015) Desenvolver mais estudos relacionando o gerenciamento de riscos com a performance da cadeia de suprimentos em questão.

Fonte: elaboração dos autores 


\section{Conclusões}

Esta pesquisa analisou 248 publicações sobre SCRM nas bases de dados Scopus, Web of Science, Science Direct, Emerald Insight e Ingenta Connect utilizando o método de pesquisa Research Profiling (Perfil de Pesquisa), por meio do software VantagePoint ${ }^{\circledR}$. A delimitação da pesquisa se deu por artigos de periódicos de língua inglesa, publicados até o final de 2015. Os dados foram extraídos em março de 2016 e, dos 543 trabalhos inicialmente obtidos (vide Tabela 1), 295 foram descartados por estarem duplicados nas bases de dados consultadas.

Ao responder as questões, (i) Quem são os autores mais produtivos?; (ii) Quais são os periódicos que mais publicam artigos na área estudada?; (iii) Quais as instituições mais produtivas?; (iv) Quais os países mais representativos na produção da área?; (v) Quem são os autores mais referenciados?; (vi) Quais são os periódicos mais referenciados?; (vii) Em quais anos houve maior número de citações?; (viii) Quando houve maior volume de publicações na área em questão?; e (ix) Quais as palavras-chave mais utilizadas no campo de pesquisa alvo?, este trabalho atingiu seu objetivo principal, que era mapear o campo de pesquisas na área de SCRM.

Espera-se que os resultados aqui apresentados poupem tempo e contribuam para avanços na pesquisa e na aprendizagem sobre SCRM, à medida em que, por exemplo:

- Sabendo que a instituição que mais publica sobre o assunto é a University of North Texas, interessados sobre o tema podem buscar desenvolver projetos de pesquisa nessa universidade;

- Sabendo que os autores que mais publicam são Backhurst (com 6 publicações); Samvedi (com 5 publicações); Wagner (4), Ekwall (4), Khan (4), Manuj (4) e Olson (4), estudantes sobre o assunto podem buscar orientações e/ou parcerias com esses autores;

- Sabendo que as palavras-chave mais utilizadas para representar o tema são "Supply Chain Risk Management", "Risk Management" e "Supply Chain Management", pesquisadores sobre SCRM possam usá-las em suas buscas nas bases de dados;

- Sabendo que as revistas que mais publicam são International Journal of Production Economics, International Journal of Production Research e Supply Chain Management: An International Journal, empresas que tenham interesse em buscar conhecimento sobre SCRM podem iniciar suas pesquisas por esses periódicos;
- Sabendo que os autores mais citados são, nessa ordem, Christopher, Tang, Chopra e Zsidisin, pesquisadores podem, ao desenvolverem pesquisas sobre SCRM, ler e analisar preliminarmente as pesquisas deles;

- Sabendo que dos 248 artigos levantados em cinco bases de dados, cerca de $95 \%$ estão na Scopus e/ou Web of Science, interessados sobre $\mathrm{o}$ assunto podem concentrar suas pesquisas nessas bases.

Além desses resultados, observou-se que: (i) $75 \%$ das publicações sobre SCRM estão concentradas nos últimos 5 anos (2011-2015); (ii) os autores mais citados não são aqueles que mais publicam sobre o assunto; (iii) os 3 periódicos mais citados são, juntos, responsáveis por quase um terço de todas as citações (ao se analisar os 20 mais citados); (iv) os países que mais publicam são Estados Unidos da América, com 69 publicações, e China, com 37 publicações - esses dois países, juntos, são responsáveis por mais de $40 \%$ das publicações sobre SCRM.

Outra contribuição relevante foi o desenvolvimento da Tabela 12, que extraiu de parte dos artigos analisados as recomendações para a realização de pesquisas futuras. Apesar dessa etapa da pesquisa ser uma revisão bibliográfica e não um Research Profiling, julgou-se pertinente e oportuno a sua elaboração, pois a intenção deste trabalho é contribuir para o desenvolvimento de pesquisas sobre SCRM. A Tabela 12 tem por finalidade auxiliar pesquisadores que estejam interessados em pesquisar sobre o tema e não possuem um ponto de partida para iniciar seu trabalho.

Durante a revisão bibliográfica constatou-se uma falta de consenso entre 23 autores que abordaram as etapas do SCRM em suas pesquisas. Alguns pesquisadores descreveram 3 etapas para o SCRM, enquanto outros abordaram a realização do SCRM em até 7 etapas. Devido a essa falta de padronização, o Quadro 1 aponta 13 "diferentes" etapas para a realização do SCRM. Ressalta-se, no entanto, que após estudos mais aprofundados sobre o conteúdo dos artigos desses autores, considerando como cada artigo definiu cada etapa proposta, ser possível harmonizar esses 13 passos em um número menor. Para corroborar ou refutar esse pressuposto, recomenda-se que esse seja um dos tópico para pesquisas futuras em SCRM.

Finalmente, com base na análise dos 248 artigos pesquisados, espera-se que futuros estudos de SCRM focalizem melhores práticas de gerenciamento de riscos, com processos de trabalho bem definidos, claramente explicados e detalhados para fins de aplicação gerencial. 


\section{Referências}

Aloini, D., Dulmin, R., Mininno, V., \& Ponticelli, S. (2012). Supply chain management: a review of implementation risks in the construction industry. Business Process Management Journal, 18(5), 735-761. http://dx.doi. org/10.1108/14637151211270135.

Autry, C. W., \& Bobbitt, L. M. (2008). Supply chain security orientation: conceptual development and a proposed framework. The International Journal of Logistics Management, 19(1), 42-64. http://dx.doi. org/10.1108/09574090810872596.

Benedek, G., Lublóy, Á., \& Vastag, G. (2014). The importance of social embeddedness: churn models at mobile providers. Decision Sciences, 45(1), 175-201. http://dx.doi.org/10.1111/deci.12057.

Blome, C., \& Schoenherr, T. (2011). Supply chain risk management in financial crises: a multiple casestudy approach. International Journal of Production Economics, 134(1), 43-57. http://dx.doi.org/10.1016/j. ijpe.2011.01.002.

Blos, M. F., Quaddus, M., Wee, H. M., \& Watanabe, K. (2009). Supply chain risk management (SCRM): a case study on the automotive and electronic industries in Brazil. Supply Chain Management: An International Journal, 14(4), 247-252. http://dx.doi.org/10.1108/13598540910970072.

Cantor, D. E., Blackhurst, J., Pan, M., \& Crum, M. (2014a). Examining the role of stakeholder pressure and knowledge management on supply chain risk and demand responsiveness. International Journal of Logistics Management, 25(1), 202-223. http://dx.doi. org/10.1108/IJLM-10-2012-0111.

Cantor, D. E., Blackhurst, J. V., \& Cortes, J. D. (2014b). The clock is ticking: the role of uncertainty, regulatory focus, and level of risk on supply chain disruption decision making behavior. Transportation Research Part E, Logistics and Transportation Review, 72, 159-172. http://dx.doi.org/10.1016/j.tre.2014.10.007.

Chae, B. K. (2015). Insights from hashtag \#supplychain and Twitter analytics: considering Twitter and Twitter data for supply chain practice and research. International Journal of Production Economics, 165, 247-259. http:// dx.doi.org/10.1016/j.ijpe.2014.12.037.

Chan, F. T. S., Samvedi, A., \& Chung, S. H. (2015). Fuzzy time series forecasting for supply chain disruptions. Industrial Management \& Data Systems, 115(3), 419435. http://dx.doi.org/10.1108/IMDS-07-2014-0199.

Chen, P. S., \& Wu, M. T. (2013). A modified failure mode and effects analysis method for supplier selection problems in the supply chain risk environment: A case study. Computers \& Industrial Engineering, 66(4), 634-642. http://dx.doi.org/10.1016/j.cie.2013.09.018.

Christopher, M., \& Lee, H. (2004). Mitigating supply chain risk through improved confidence. International Journal of Physical Distribution \& Logistics Management, 34(5), 388-396. http://dx.doi.org/10.1108/09600030410545436.
Christopher, M., Mena, C., Khan, O., \& Yurt, O. (2011). Approaches to managing global sourcing risk. Supply Chain Management: An International Journal, 16(2), 67-81. http://dx.doi.org/10.1108/13598541111115338.

Cohen, M. A., \& Kunreuther, H. (2007). Operations risk management: overview of Paul Kleindorfer's contributions. Production and Operations Management, 16(5), 525-541.

Colicchia, C., Dallari, F., \& Melacini, M. (2010). Increasing supply chain resilience in a global sourcing context. Production Planning and Control, 21(7), 680-694. http://dx.doi.org/10.1080/09537280903551969.

Colicchia, C., \& Strozzi, F. (2012). Supply chain risk management: a new methodology for a systematic literature review. Supply Chain Management: An International Journal, 17(4), 403-418. http://dx.doi. org/10.1108/13598541211246558.

Dani, S., \& Deep, A. (2010). Fragile food supply chains: reacting to risks. International Journal of Logistics: Research and Applications, 13(5), 395-410. http:// dx.doi.org/10.1080/13675567.2010.518564.

Davarzani, H., Farahani, R. Z., \& Rahmandad, H. (2015). Understanding econo-political risks: impact of sanctions on an automotive supply chain. International Journal of Operations \& Production Management, 35(11), $1567-$ 1591. http://dx.doi.org/10.1108/IJOPM-01-2013-0021.

Diabat, A., Govindan, K., \& Panicker, V. V. (2012). Supply chain risk management and its mitigation in a food industry. International Journal of Production Research, 50(11), 3039-3050. http://dx.doi.org/10.1080/0020754 3.2011 .588619 .

Eldridge, J. (2006). Data visualization tools: a perspective from the pharmaceutical industry. World Patent Information, 28(1), 43-49. http://dx.doi.org/10.1016/j. wpi.2005.10.007.

Ekwall, D., \& Lantz, B. (2013). Seasonality of cargo theft at transport chain locations. International Journal of Physical Distribution \& Logistics Management, 43(9), 728-746. http://dx.doi.org/10.1108/IJPDLM-06-2012-0175.

Faisal, M. N., Banwet, D. K., \& Shankar, R. (2006). Supply chain risk mitigation: modeling the enablers. Business Process Management Journal, 12(4), 535-552. http:// dx.doi.org/10.1108/14637150610678113.

Finch, P. (2004). Supply chain risk management. Supply Chain Management: An International Journal, 9(2), 183-196. http://dx.doi.org/10.1108/13598540410527079.

Gaonkar, R. S., \& Viswanadham, N. (2007). Analytical framework for the management of risk in supply chains. IEEE Transactions on Automation Science and Engineering, 4(2), 265-273. http://dx.doi.org/10.1109/ TASE.2006.880540.

Ghadge, A., Dani, S., \& Kalawsky, R. (2012). Supply chain risk management: present and future scope. The International Journal of Logistics Management, 23(3), 313-339. http://dx.doi.org/10.1108/09574091211289200. 
Ghadge, A., Dani, S., Chester, M., \& Kalawsky, R. (2013). A systems approach for modelling supply chain risks. Supply Chain Management: An International Journal, 18(5), 523-538. http://dx.doi.org/10.1108/SCM-112012-0366.

Giannakis, M., \& Louis, M. (2011). A multi-agent based framework for supply chain risk management. Journal of Purchasing and Supply Management, 17(1), 23-31. http://dx.doi.org/10.1016/j.pursup.2010.05.001.

Goh, M., Lim, J. Y. S., \& Meng, F. (2007). A stochastic model for risk management in global supply chain networks. European Journal of Operational Research, 182(1), 164-173. http://dx.doi.org/10.1016/j.ejor.2006.08.028.

Golgeci, I., \& Ponomarov, S. Y. (2013). Does firm innovativeness enable effective responses to supply chain disruptions? an empirical study. Supply Chain Management: An International Journal, 18(6), 604617. http://dx.doi.org/10.1108/SCM-10-2012-0331.

Govindan, K., Azevedo, S. G., Carvalho, H., \& Cruz-Machado, V. (2014). Impact of supply chain management practices on sustainability. Journal of Cleaner Production, 85, 212-225. http://dx.doi.org/10.1016/j.jclepro.2014.05.068.

Grötsch, V. M., Blome, C., \& Schleper, M. C. (2013). Antecedents of proactive supply chain risk management: a contingency theory perspective. International Journal of Production Research, 51(10), 2842-2867. http:// dx.doi.org/10.1080/00207543.2012.746796.

Guo, Y. (2011). Research on knowledge-oriented supply chain risk management system model. Journal of Management and Strategy, 2(2), 72-77. http://dx.doi. org/10.5430/jms.v2n2p72.

Hallikas, J., Karvonen, I., Pulkkinen, U., Virolainen, V., \& Tuominen, M. (2004). Risk management processes in supplier networks. International Journal of Production Economics, 90(1), 47-58. http://dx.doi.org/10.1016/j. ijpe.2004.02.007.

Harland, C., Brenchley, R., \& Walker, H. (2003). Risk in supply networks. Journal of Purchasing and Supply Management, 9(2), 51-62. http://dx.doi.org/10.1016/ S1478-4092(03)00004-9.

Hendricks, K. B., Singhal, V. R., \& Zhang, R. R. (2009). The effect of operational slack, diversification, and vertical relatedness on the stock market reaction to supply chain disruptions. Journal of Operations Management, 27(3), 233-246. http://dx.doi.org/10.1016/j.jom.2008.09.001.

Hofmann, H., Busse, C., Bode, C., \& Henke, M. (2014). Sustainability-related supply chain risks: conceptualization and management. Business Strategy and the Environment, 23(3), 160-172. http://dx.doi.org/10.1002/bse.1778.

Hult, G. T. M., Craighead, C. W., \& Ketchen, D. J. Jr (2010). Risk Uncertainty and supply chain decisions: a real options perspective. Decision Sciences, 41(3), 435-458. http://dx.doi.org/10.1111/j.1540-5915.2010.00276.x.
Islam, N., \& Miyazaki, K. (2010). An empirical analysis of nanotechnology research domains. Technovation, 30(4), 229237. http://dx.doi.org/10.1016/j.technovation.2009.10.002.

Jia, F., \& Rutherford, C. (2010). Mitigation of supply chain relational risk caused by cultural differences between China and the West. The International Journal of Logistics Management, 21(2), 251-270. http://dx.doi. org/10.1108/09574091011071942.

Jüttner, U., Peck, H., \& Christopher, M. (2003). Supply chain risk management: outlining an agenda for future research. International Journal of Logistics: Research \& Applications, 6(4), 197-210. http://dx.doi.org/10.10 $80 / 13675560310001627016$.

Jüttner, U., \& Maklan, S. (2011). Supply chain resilience in the global financial crisis: an empirical study. Supply Chain Management: An International Journal, 16(4), 246-259. http://dx.doi.org/10.1108/13598541111139062.

Kern, D., Moser, R., Hartmann, E., \& Moder, M. (2012). Supply risk management: model development and empirical analysis. International Journal of Physical Distribution \& Logistics Management, 42(1), 60-82. http://dx.doi.org/10.1108/09600031211202472.

Khan, O., \& Burnes, B. (2007). Risk and supply chain management: creating a research agenda. The International Journal of Logistics Management, 18(2), 197-216. http:// dx.doi.org/10.1108/09574090710816931.

Khan, O., Christopher, M., \& Creazza, A. (2012). Aligning product design with the supply chain: a case study. Supply Chain Management: An International Journal, 17(3), 323-336. http://dx.doi.org/10.1108/13598541211227144.

Kilubi, I., \& Haasis, H. D. (2015). Supply chain risk management enablers: a framework development through systematic review of the literature from 2000 to 2015. International Journal of Business Science and Applied Management, 10(1), 35-54.

Kim, J., Hwang, M., Jeong, D., \& Jung, H. (2012). Expert Systems with Applications Technology trends analysis and forecasting application based on decision tree and statistical feature analysis. Expert Systems with Applications, 39(16), 12618-12625. http://dx.doi. org/10.1016/j.eswa.2012.05.021.

Kleindorfer, P. R., \& Saad, G. H. (2005). Managing disruption risks in supply chains. Production and Operations Management, 14(1), 53-68. http://dx.doi. org/10.1111/j.1937-5956.2005.tb00009.x.

Kumar, S., \& Harrison, G. (2012). Expect the unexpected: supply chain disruption and opportunity for US companies: a business case. Transportation Journal, 51(1), 118-136.

Lavastre, O., Gunasekaran, A., \& Spalanzani, A. (2012). Supply chain risk management in French companies. Decision Support Systems, 52(4), 828-838. http://dx.doi. org/10.1016/j.dss.2011.11.017.

Li, G., Fan, H., Lee, P. K. C., \& Cheng, T. C. E. (2015). Joint supply chain risk management: an agency and collaboration perspective. International Journal of 
Production Economics, 164, 83-94. http://dx.doi. org/10.1016/j.ijpe.2015.02.021.

Liew, K. C., \& Lee, C. K. M. (2012). Modelling and risk management in the offshore and marine industry supply chain. International Journal of Engineering Business Management, 4(1), 1-7.

Lin, Y., \& Zhou, L. (2011). The impacts of product design changes on supply chain risk: a case study. International Journal of Physical Distribution \& Logistics Management, 41(2), 162-186. http://dx.doi. org/10.1108/09600031111118549.

Liu, F., Wang, J. J., Chen, H., \& Yang, D. L. (2014). Machine scheduling with outsourcing. The International Journal of Logistics Management, 25(1), 133-159. http://dx.doi. org/10.1108/IJLM-12-2012-0142.

Lockamy, A. 3rd, \& Mccormack, K. (2012). Modeling supplier risks using Bayesian networks. Industrial Management \& Data Systems, 112(2), 313-333. http:// dx.doi.org/10.1108/02635571211204317.

Manuj, I., \& Mentzer, J. T. (2008a). Global supply chain risk management strategies. International Journal of Physical Distribution \& Logistics Management, 38(3), 192-223. http://dx.doi.org/10.1108/09600030810866986.

Manuj, I., \& Mentzer, J. T. (2008b). Global supply chain risk management. Journal of Business Logistics, 29(1), 133-155. http://dx.doi.org/10.1002/j.2158-1592.2008. tb00072.x.

Manuj, I., Esper, T. L., \& Stank, T. P. (2014). Supply chain risk management approaches under different conditions of risk. Journal of Business Logistics, 35(3), 241-258. http://dx.doi.org/10.1111/jbl.12051.

Manuj, I., Mentzer, J. T., \& Bowers, M. R. (2009). Improving the rigor of discrete-event simulation in logistics and supply chain research. International Journal of Physical Distribution \& Logistics Management, 39(3), 172-201. http://dx.doi.org/10.1108/09600030910951692.

Marija, M., Ivan, B., \& Dušan, R. (2015). Supply chain risk management using software tool historical perspective of risk management. Acta Polytechnica Hungarica, 12(4), 167-182.

Matook, S., Lasch, R., \& Tamaschke, R. (2009). Supplier development with benchmarking as part of a comprehensive supplier risk management framework. International Journal of Operations \& Production Management, 29(3), 241-267. http://dx.doi.org/10.1108/01443570910938989.

Mizgier, K. J., Wagner, S. M., \& Jüttner, M. P. (2015). Disentangling diversification in supply chain networks. International Journal of Production Economics, 162, 115-124. http://dx.doi.org/10.1016/j.ijpe.2015.01.007.

Moeinzadeh, P. B., \& Hajfathaliha, A. (2009). A combined fuzzy decision making approach to supply chain risk assessment. World Academy of Science, Engineering and Technology, 60(12), 519-535.
Nagali, V., Hwang, J., Sanghera, D., Gaskins, M., Pridgen, M., Thurston, T., Mackenroth, P., Branvold, D., Scholler, P., \& Shoemaker, G. (2008). Procurement Risk Management (PRM) at Hewlett-Packard company. Interfaces, 38(1), 51-60. http://dx.doi.org/10.1287/inte.1070.0333.

Narasimhan, R., \& Talluri, S. (2009). Perspectives on risk management in supply chains. Journal of Operations Management, 27(2), 114-118. http://dx.doi.org/10.1016/j. jom.2009.02.001.

Nooraie, S. V., \& Parast, M. (2015). A multi-objective approach to supply chain risk management: integrating visibility with supply and demand risk. International Journal of Production Economics, 161, 192-200. http:// dx.doi.org/10.1016/j.ijpe.2014.12.024.

Norrman, A., \& Jansson, U. (2004). Ericsson's proactive supply chain risk management approach after a serious sub-supplier accident. International Journal of Physical Distribution \& Logistics Management, 34(5), 434-456. http://dx.doi.org/10.1108/09600030410545463.

Oehmen, J., Ziegenbein, A., Alard, R., \& Schonsleben, P. (2009). System-oriented supply chain risk management. Production Planning and Control, 20(4), 343-361. http:// dx.doi.org/10.1080/09537280902843789.

Ojha, D., \& Gokhale, R. A. (2009). Logistical business continuity planning-scale development and validation. The International Journal of Logistics Management, 20(3), 342-359. http://dx.doi.org/10.1108/09574090911002814.

Olson, D. L., \& Wu, D. (2011). Risk management models for supply chain: a scenario analysis of outsourcing to China. Supply Chain Management: An International Journal, 16(6), 401-408. http://dx.doi. org/10.1108/13598541111171110.

O’Kane, J. F., Spenceley, J. R., \& Taylor, R. (2000). Simulation as an essential tool for advanced manufacturing technology problems. Journal of Materials Processing Technology, 107(1-3), 412-424.

Porter, A. L., Kongthon, A., \& Lu, C. (2002). Research profiling: improving the literature review. Scientometrics, 53(3), 351-370. http://dx.doi.org/10.1023/A:1014873029258.

Peng, M., Peng, Y., \& Chen, H. (2014). Post-seismic supply chain risk management: a system dynamics disruption analysis approach for inventory and logistics planning. Computers \& Operations Research, 42, 14-24. http:// dx.doi.org/10.1016/j.cor.2013.03.003.

Pujawan, I. N., \& Geraldin, L. H. (2009). House of risk: a model for proactive supply chain risk management. Business Process Management Journal, 15(6), 953967. http://dx.doi.org/10.1108/14637150911003801.

Rajesh, R., \& Ravi, V. (2015). Modeling enablers of supply chain risk mitigation in electronic supply chains: a Grey-DEMATEL approach. Computers \& Industrial Engineering, 87, 126-139. http://dx.doi.org/10.1016/j. cie.2015.04.028.

Rangel, D. A., Oliveira, T. K., \& Leite, M. S. A. (2015). Supply chain risk classification: discussion and proposal. 
International Journal of Production Research, 53(22), 6868-6887. http://dx.doi.org/10.1080/00207543.2014 .910620 .

Ritchie, B., \& Brindley, C. (2007). An emergent frameworkfor supply chain risk management and performance measure. The Journal of the Operational Research Society, 58(11), 1398-1411. http://dx.doi.org/10.1057/ palgrave.jors.2602412.

Ruiz-Torres, A. J., Mahmoodi, F., \& Zeng, A. Z. (2013). Supplier selection model with contingency planning for supplier failures. Computers \& Industrial Engineering, 66(2), 374-382. http://dx.doi.org/10.1016/j.cie.2013.06.021.

Saaty, T. L. (1994). How to make a decision: the analytic hierarchy process. Interfaces, 24(6), 19-43.

Samvedi, A., Jain, V., \& Chan, F. T. S. (2013). Quantifying risks in a supply chain through integration of fuzzy AHP and fuzzy TOPSIS. International Journal of Production Research, 51(8), 2433-2442. http://dx.doi.org/10.1080 /00207543.2012.741330.

Sawik, T. (2011a). Selection of a dynamic supply portfolio in make-to-order environment withrisks. Computers \& Operations Research, 38(4), 782-796. http://dx.doi. org/10.1016/j.cor.2010.09.011.

Sawik, T. (2011b). Supplier selection in make-to-order environment with risks. Mathematical and Computer Modelling, 53(9-10), 1670-1679. http://dx.doi. org/10.1016/j.mcm.2010.12.039.

Schmitt, A. J., \& Singh, M. (2012). A quantitative analysis of disruption risk in a multi-echelon supply chain. International Journal of Production Economics, 139(1), 22-32. http://dx.doi.org/10.1016/j.ijpe.2012.01.004.

Schmitt, A. J., \& Snyder, L. V. (2012). Infinite-horizon models for inventory control under yield uncertainty and disruptions. Computers \& Operations Research, 39(4), 850-862. http://dx.doi.org/10.1016/j.cor.2010.08.004.

Singhal, P., Agarwal, G., \& Mittal, M. L. (2011). Supply chain risk management : review, classification and future research directions. Int. Journal of Business Science and Applied Management, 6(3), 15-42.

Sodhi, M. S., Son, B., \& Tang, C. (2012). Researchers' perspectives on supply chain risk management. Production and Operations Management, 21(1), 1-13. http://dx.doi. org/10.1111/j.1937-5956.2011.01251.x.

Sodhi, M. S., \& Tang, C. S. (2009). Modeling supply-chain planning under demand uncertainty using stochastic programming: A survey motivated by asset-liability management. International Journal of Production Economics, 121(2), 728-738. http://dx.doi.org/10.1016/j. ijpe.2009.02.009.

Son, J. Y., \& Orchard, R. K. (2013). Effectiveness of policies for mitigating supply disruptions. International Journal of Physical Distribution \& Logistics Management, 43(8), 684-706. http://dx.doi.org/10.1108/IJPDLM-04-2012-0109.
Sun, J., Matsui, M., \& Yin, Y. (2012). Supplier risk management: An economic model of P-chart considered due-date and quality risks. International Journal of Production Economics, 139(1), 58-64. http://dx.doi. org/10.1016/j.ijpe.2012.03.004.

Tang, C. S. (2006). Perspectives in supply chain risk management. International Journal of Production Economics, 103(2), 451-488. http://dx.doi.org/10.1016/j. ijpe.2005.12.006.

Tang, O., \& Musa, S. N. (2011). Identifying risk issues and research advancements in supply chain risk management. International Journal of Production Economics, 133(1), 25-34.

Tang, O., Musa, S. N., \& Li, J. (2012). Dynamic pricing in the newsvendor problem with yield risks. International Journal of Production Economics, 139(1), 127-134. http://dx.doi.org/10.1016/j.ijpe.2011.01.018.

Thun, J. H., \& Hoenig, D. (2011). An empirical analysis of supply chain risk management in the German automotive industry. International Journal of Production Economics, 131(1), 242-249. http://dx.doi.org/10.1016/j. ijpe.2009.10.010.

Thun, J., Drüke, M., \& Hoenig, D. (2011). Managing uncertainty: an empirical analysis of supply chain risk management in small and medium-sized enterprises. International Journal of Production Research, 49(18), 5511-5525. http://dx.doi.org/10.1080/00207543.201 1.563901 .

Trkman, P., \& McCormack, K. (2009). Supply chain risk in turbulent environments: a conceptual model for managing supply chain network risk. International Journal of Production Economics, 119(2), 247-258. http://dx.doi.org/10.1016/j.ijpe.2009.03.002.

Tse, Y. K., Tan, K. H., Chung, S. H., \& Lim, M. K. (2011). Quality risk in global supply network. Journal of Manufacturing Technology Management, 22(8), 10021013. http://dx.doi.org/10.1108/17410381111177458.

Tummala, R., \& Schoenherr, T. (2011). Assessing and managing risks using the Supply Chain Risk Management Process (SCRMP). Supply Chain Management: An International Journal, 16(6), 474-483. http://dx.doi. org/10.1108/13598541111171165.

Tuncel, G., \& Alpan, G. (2010). Risk assessment and management for supply chain networks: a case study. Computers in Industry, 61(3), 250-259. http://dx.doi. org/10.1016/j.compind.2009.09.008.

Urciuoli, L., Mohanty, S., Hintsa, F., \& Boekesteijn, E. G. (2014). The resilience of energy supply chains: a multiple case study approach on oil and gas supply chains to Europe. Supply Chain Management: An International Journal, 19(1), 46-63. http://dx.doi.org/10.1108/SCM09-2012-0307.

Vilko, J. P. P., \& Hallikas, J. M. (2012). Risk assessment in multimodal supply chains. International Journal of Production Economics, 140(2), 586-595. http://dx.doi. org/10.1016/j.ijpe.2011.09.010. 
Vilko, J., Ritala, P., \& Edelmann, J. (2014). On uncertainty in supply chain risk management. International Journal of Logistics Management, 25(1), 3-19. http://dx.doi. org/10.1108/IJLM-10-2012-0126.

Wagner, S. M., \& Neshat, N. (2012). A comparison of supply chain vulnerability indices for different categories of firms. International Journal of Production Research, 50(11), 2877-2891. http://dx.doi.org/10.1080/002075 43.2011.561540.

Wever, M., Wognum, N., Trienekens, J., \& Omta, O. (2012). Managing transaction risks in interdependent supply chains: an extended transaction cost economics perspective. Journal on Chain and Network Science, 12(3), 243-260. http://dx.doi.org/10.3920/JCNS2012.x214.

Wieland, A., \& Wallenburg, C. M. (2012). Dealing with supply chain risks: linking risk management practices and strategies to performance. International Journal of Physical Distribution \& Logistics Management, 42(10), 887-905. http://dx.doi.org/10.1108/09600031211281411.

Williams, Z., Lueg, J. E., \& Lemay, S. A. (2008). Supply chain security : an overview and research agenda. The International Journal of Logistic Management, 19(2), 254-281. http://dx.doi.org/10.1108/09574090810895988.

Wu, D. S., \& Olson, D. L. (2008). Supply chain risk, simulation, and vendor selection. International Journal of Production Economics, 114(2), 646-655. http://dx.doi. org/10.1016/j.ijpe.2008.02.013.

Wu, T., Blackhurst, J., \& Chidambaram, V. (2006). A model for inbound supply risk analysis. Computers in Industry, 57(4), 350-365. http://dx.doi.org/10.1016/j. compind.2005.11.001.

Wu, T., Huang, S., Blackhurst, J., Zhang, X., \& Wang, S. (2013). Supply chain risk management: an agent-based simulation to study the impact of retail stockouts. IEEE Transactions on Engineering Management, 60(4), 676686. http://dx.doi.org/10.1109/TEM.2012.2190986.

Yang, B., \& Yang, Y. (2010). Postponement in supply chain risk management: a complexity perspective. International Journal of Production Research, 48(7), 1901-1912. http://dx.doi.org/10.1080/00207540902791850.

Yu, Y., Xiong, W., \& Cao, Y. (2015). A conceptual model of Supply Chain Risk Mitigation: the role of supply chain integration and organizational risk propensity. Journal of Coastal Research, 73, 95-98. http://dx.doi. org/10.2112/SI73-017.1.

Zsidisin, G., Ragatz, G. L., \& Melnyk, S. A. (2005). An institutional theory perspective of business continuity planning for purchasing and supply management. International Journal of Production Research, 43(16), 3401-3420. http://dx.doi.org/10.1080/00207540500095613. 